\title{
Local stability of Evolutionary Attractors for continuous structured populations.
}

\author{
Gaël Raoul \\ CMLA, ENS Cachan, CNRS, PRES UniverSud, 61, avenue du Président Wilson, 94235 Cachan Cedex, France \\ e-mail: raoul@cmla.ens-cachan.fr
}

January 8, 2009

\begin{abstract}
In this paper, we are interested in the nonlinear stability of Dirac-type steady solutions to an integro-differential equation appearing in the study of populations which are structured with respect to a quantative (continuous) trait. We show that stability conditions of adaptive dynamics extend to this model.
\end{abstract}

\section{Introduction}

Adaptive dynamics (see [10], [11], [7], [8]) is a branch of evolutionary ecology, that aims at describing the Darwinian evolution of populations along a phenotypic trait, which characterises each individual. This trait as well as the state of the resident population define the survival rate and reproduction rate of the individual. In the asexual case that we consider here, an offspring has exactly the same trait as its ancestor, except if a mutation occurs. Adaptive dynamics provides in particular some conditions for a population of a given trait to be stable with respect to evolution. Those stable populations are then called Evolutionary attractors.

We consider in this article a simple model to describe a population which is structured with respect to a quantitative continuous trait. We represent the one-dimensional phenotypic trait as $x \in X \subset \mathbb{R}$, and the population (at a given time $t \geq 0$ ) by a measure $g(t, \cdot)$ over the set of phenotypic traits $X$. Then, the population evolution is described by the following integrodifferential equation:

$$
\frac{\partial g}{\partial t}=s_{g} g,
$$

where $s_{g}$ is the fitness, that is the birth rate minus the death rate. We neglect here the mutations, since they do not play an important role when the initial condition is strictly positive, which we assume here (see [4]). In this paperw we only consider logistic fitnesses of the following type:

$$
s_{g}(x)=a(x)-\int_{X} b(x, y) d g(y),
$$

where $a: X \rightarrow \mathbb{R}$ represents the fitness without competition, and $b: X \times X \rightarrow \mathbb{R}_{+}$represents the part part of fitness due to competition. 
This model has been derived from stochastic models of finite populations (see [3]), taking the limit of an infinite number of individuals with the correct time scale, with an additionnal mutation term. It has also been studied in [4], where numerical simulations show some speciation processes: from an initial population where every traits are present, a finite number of traits are selected, while the others become extinct.

In this article, we complete the study of [4] concerning the large time behaviour of (1.1). According to Adaptive dynamics, the population should tend to an Evolutionary Attractor of the selection process $s_{g}$ (see [5]). However, while Evolutionary Attractors are well defined for monomorphic populations (see [5]), the case of several coexisting traits remains unclear. In the first part of this paper, we propose therefore an extension of the consept of evolutionary Attractors to the non-monomorphic case. Then, we show that with this definition, Evolutionary Attractors are locally stable for our model (1.1). In a last section, we study numerically some examples.

In section 2, we give definitions which enable to extend to non-monomorphic populations some of the concepts of adaptive dynamics. Then, section 3 is devoted to the presentation of results already obtained for the model that we consider, and to the exposition of the main theorem. This theorem is proven in section 4 , and numerical simulations are presented in section 5 .

\section{Adaptive dynamics in the case of non-monomorphic popula- tions.}

Our results will be obtained under the following

Assumption 1: $X$ is a compact interval of $\mathbb{R}, a \in C^{2}(X), b \in C^{1}(X, X), \forall y \in X, b(\cdot, y) \in$ $C^{2}(X)$, and:

$$
\begin{array}{r}
a, a^{\prime}, a^{\prime \prime} \in L^{\infty}(X), \\
b, \partial_{x} b, \partial_{x x}^{2} b \in L^{\infty}(X, X) .
\end{array}
$$

Note that for a population consisting in a finite number of species $g(t)=\sum_{i=1}^{n} \rho_{i}(t) \delta_{\bar{x}_{i}}$, eq. (1.1) becomes:

$$
\forall i=1, \ldots, n, \quad \rho_{i}^{\prime}(t)=\left[a\left(\bar{x}_{i}\right)-\sum_{j=1}^{n} \rho_{j}(t) b\left(\bar{x}_{i}, \bar{x}_{j}\right)\right] \rho_{i}(t) .
$$

Definition 2.1 Let $\left(\bar{x}_{i}\right)_{i=1, \ldots, n} \in X^{n}$. We assume that the system of ordinary differential equations (2.3) admits a steady solution $\left(\bar{\rho}_{i}\right)_{i=1, \ldots, n},\left(\bar{\rho}_{i} \neq 0 \forall i=1, \ldots, n\right)$, which is linearly stable, that is:

$$
\begin{gathered}
\left(b\left(\bar{x}_{i}, \bar{x}_{j}\right)\right)_{i, j=1, \ldots, n} \text { is invertible, } \\
\mathcal{R} e\left[\operatorname{Spec}\left(D F_{\left.\left(\bar{\rho}_{i}\right)_{i=1, \ldots, n}\right)}\right) \subset \mathbb{R}_{-}^{*},\right.
\end{gathered}
$$

where $F\left(\left(\rho_{i}\right)_{i=1, \ldots, n}\right):=\left(\left[a\left(\bar{x}_{i}\right)-\sum_{j=1}^{n} \rho_{j} b\left(\bar{x}_{i}, \bar{x}_{j}\right)\right] \rho_{i}\right)_{i=1, \ldots, n}$. 
Such a familly $\left(\bar{x}_{i}\right)_{i=1, \ldots, n} \in X^{n}$ is called a strategy. It means that the population $g=$ $\sum_{i=1}^{n} \bar{\rho}_{i} \delta_{\bar{x}_{i}}$, where

$$
\left(\bar{\rho}_{i}\right)_{i=1, \ldots, n}=\left(b\left(\bar{x}_{i}, \bar{x}_{j}\right)\right)_{i, j=1, \ldots, n}^{-1}\left(a\left(\bar{x}_{i}\right)\right)_{i=1, \ldots, n},
$$

is stable as long as no over trait is present (that is it is ecologically stable).

We now define Evolutionary stable strategies (ESS), and Evolutionary Attractors (see [5], [8], [10], [7]): They are steady solutions of eq. (1.1) which have a stronger stability than strategies.

For a discution on ESS and Evolutionary Attractors, and numerical examples, see Section 4.

Definition 2.2 We suppose that Assumption 1 holds. A strategy $\left(\bar{x}_{i}\right)_{i=1, \ldots, n}$ is called ESS (Evolutionary Stable Strategy) if:

$$
\begin{aligned}
& \forall i=1, \ldots, n, \quad\left(\partial_{x} s_{\sum_{j=1}^{n} \bar{\rho}_{j} \delta_{\bar{x}_{j}}}\right)\left(\bar{x}_{i}\right)=0, \\
& \forall i=1, \ldots, n, \quad\left(\partial_{x x}^{2} s_{\sum_{j=1}^{n} \bar{\rho}_{j} \delta_{\bar{x}_{j}}}\right)\left(\bar{x}_{i}\right)<0,
\end{aligned}
$$

where $\bar{\rho}_{j}$ is defined in (2.6), and $\partial_{x} s_{\sum_{j=1}^{n} \bar{\rho}_{j} \delta_{\bar{x}_{j}}}, \partial_{x x}^{2} s_{\sum_{j=1}^{n} \bar{\rho}_{j} \delta_{\bar{x}_{j}}}$ are derivatives of $x \mapsto s_{\sum_{j=1}^{n} \bar{\rho}_{j} \delta_{\bar{x}_{j}}}(x)=$ $a(x)-\sum_{j=1}^{n} \bar{\rho}_{j} b\left(x, \bar{x}_{j}\right)$.

Remark 2.3 If the first condition is true, the stategy is called singular (see [10]).

Definition 2.4 Let $G$ be defined by:

$$
G:\left(x_{i}\right)_{i=1, \ldots, n} \mapsto\left(\partial_{x} s_{\sum_{j=1}^{n} \rho_{j} \delta_{x_{j}}}\left(x_{i}\right)\right)_{i=1, \ldots, n},
$$

where $\rho_{j}=\left(b\left(x_{i}, x_{j}\right)\right)_{i, j=1, \ldots, n}^{-1}\left(a\left(x_{i}\right)\right)_{i=1, \ldots, n}$.

A strategy $\left(\bar{x}_{i}\right)_{i=1, \ldots, n}$ is an Evolutionary Attractor if it is an ESS, and if there exists $\nu>0$ such that :

$$
\forall u \in \mathbb{R}^{n}, \quad{ }^{t} u \operatorname{diag}\left(\left(\frac{1}{-\partial_{x x}^{2} s_{\sum_{j=1}^{n} \bar{\rho}_{j} \delta_{\bar{x}_{j}}}\left(\bar{x}_{i}\right)}\right)_{i=1, \ldots, n}\right) D G\left(\left(\bar{x}_{i}\right)_{i=1, \ldots, n}\right) u<-\nu\|u\|^{2} .
$$

Remark 2.5 In adaptive dynamics, where we consider a monomorphic population $g(t, x)=$ $\rho(t) \delta_{x(t)}$, the motion of $x(\cdot)$ is given by the so-called canonical equation (see [8], [2]):

$$
\frac{d}{d t} x(t)=C\left(\rho(t), \epsilon, \partial_{x x}^{2} s_{\rho(t) \delta_{x(t)}}(x(t))\right) \partial_{x} s_{\rho(t) \delta_{x(t)}}(x(t)), \rho(t):=\frac{a(x(t))}{b(0)},
$$

where $\epsilon$ describes the frequency of mutations. One then defines Convergent stable strategies $(C S S)$ as strategies $\bar{x}$ stable for this ordinary differential equation. Evolutionary Attractors are then strategies that are both ESS and CSS. Our definition can be seen as an extension of this definition when several traits coexist : ineq. (2.8) means that the $L^{2}$ norm is a Lyapounov function for the following ordinary differential equation :

$$
\forall i=1, \ldots, n, \quad \frac{d}{d t} x_{i}=\frac{1}{-\partial_{x x}^{2} s_{\sum_{j=1}^{n} \bar{\rho}_{j} \delta_{\bar{x}_{j}}}\left(\bar{x}_{i}\right)} \partial_{x} s \sum_{j=1}^{n} \rho_{j} \delta_{x_{j}}\left(x_{i}\right)
$$

where $\left(\rho_{j}\right)_{j=1, \ldots, n}=\left(b\left(x_{i}, x_{j}\right)\right)_{i, j=1, \ldots, n}^{-1}\left(a\left(x_{i}\right)\right)_{i=1, \ldots, n}$. 


\section{Continuously structured populations}

In this section, we shall present an extension of the model (2.3) to the case of a population $g(t, \cdot) \in M^{1}(X)$ which may contain an infinite (continuous) number of traits.

\subsection{The Model and existing results.}

If we neglect mutations, a population $g: t \in \mathbb{R}_{+} \mapsto M^{1}(X)$ submitted to a logistic competition will evolve under the effect of fitness as follows:

$$
\left\{\begin{array}{l}
g(0, \cdot)=g_{0} \in M^{1}(X) \\
\partial_{t} g(t, x)=\left[a(x)-\int_{X} b(x, y) g(t, y) d y\right] g(t, x)
\end{array}\right.
$$

This model has been studied in [4]. We recall here the main result of that paper.

Theorem 3.6 (Theorems 2.1 and 3.1 in [4].)

Let $X$ be a compact interval of $\mathbb{R}, g_{0} \in L^{1}(X), g_{0} \leq 0$ be the initial population, and $a, b \in$ $C^{1}(X)$ such that:

$$
\{x ; a(x)>0\} \neq \emptyset \text { and } \forall x, y \in \operatorname{supp}\left(g_{0}\right), 0<b_{m} \leq b(x, y) \leq b_{M}<\infty,
$$

(where supp $\left(g_{0}\right)$ denotes the support of the function $\left.g_{0}\right)$. Then, there exists a unique solution $g \in C\left(\left[0,+\infty\left[; L^{1}(X)\right)\right.\right.$ of eq. (3.9). If a is bounded, $g$ is bounded with respect to time:

$$
\|g(t, \cdot)\|_{L^{1}(X)} \leq \max \left(\left\|g_{0}\right\|_{L^{1}(X)}, \frac{\sup _{X} a}{b_{m}}\right) .
$$

Let us define $f_{\varepsilon}(t, x):=g\left(\frac{t}{\varepsilon}, x\right)$. For each sequence $\varepsilon_{n} \rightarrow 0$, there exists a subsequence (still denoted by $\left.\left(\varepsilon_{n}\right)_{n}\right)$ such that $\left(f_{\varepsilon_{n}}\right)$ converges to a limit $f$ which is a measure with respect to $y$ :

$$
f_{\varepsilon_{n}} \underset{n \rightarrow \infty}{\longrightarrow} f \quad L^{\infty}(w *] 0, T\left[, \sigma\left(M^{1}(X), C_{b}\right)\right) .
$$

Moreover, if we define

$$
R_{\varepsilon}(t, x):=\int_{0}^{t}\left(a(x)-\int_{X} b(x, y) f_{\varepsilon}(\sigma, y) d y\right) d \sigma
$$

and

$$
R(t, x):=\int_{0}^{t}\left(a(x)-\int_{X} b(x, y) f(\sigma, y) d y\right) d \sigma
$$

then:

- If a,b satisfy Assumption $1, R$ is differentiable with respect to $t$, and is $C^{2}$ with respect to $x$

- $R_{\varepsilon}$ converges to $R$ uniformly on each compact set of $\mathbb{R}_{+} \times X$, 
- for any $t \geq 0, R(t, x) \leq 0$ for all $x \in X$ s.t. $g_{0}(x)>0$,

- $\operatorname{supp}(f(t, \cdot)) \subset\{x \in X ; R(t, x)=0\}$.

Remark 3.7 As a consequence, still under Assumption 1, provided that $R(t, x)=0$,

If $x \in \operatorname{int}\left(\operatorname{supp}\left(g_{0}\right)\right)$, then $\partial_{x} R(t, x)=0$ and $\partial_{x x}^{2} R(t, x) \leq 0$.

if $x \in \partial\left(\operatorname{supp}\left(g_{0}\right)\right)$, then $\nu \partial_{x} R(t, x) \leq 0$, where $\nu \in\{1,-1\}$ is the outer vector of int $\left(\operatorname{supp}\left(g_{0}\right)\right)$ at point $x$.

\subsection{Statement of the result: local stability of evolutionary attractors.}

In many numerical experiments, populations satisfying (3.9) seem to gather around a finite number of phenotypic traits which are Evolutionary Attractors. We also know that in general, Evolutionary Attractors are not unique (see subsection 5.2), and therefore not globally stable. The main result of this work is that the Evolutionary Attractors defined in the first section are nevertheless locally stable.

We define, for a given strategy $\left(\bar{x}_{i}\right)_{i=1, \ldots, n}$,

$$
I_{i}^{\delta}:=\left\{x ;\left|x-\bar{x}_{i}\right|<\delta\right\}
$$

The following assumption defines the type of initial condition for which our local stability results hold:

Assumption 2: For some $\delta, \lambda>0$,

- $\operatorname{supp} g_{0}=\cup_{i=1, \ldots, n} I_{i}^{\delta}$, and more precisely $g_{0} \neq 0$ a.e. on $\cup_{i=1, \ldots, n} I_{i}^{\delta}$,

- $\forall i=1, \ldots, n, \quad\left|\int_{I_{i}^{\delta}} g_{0}(x) d x-\bar{\rho}_{i}\right| \leq \lambda$.

Remark 3.8 Note that those hypothesis on $g_{0}$ are much stronger than the simpler assumption that $g$ is close to $\sum_{i=1}^{n} \bar{\rho}_{i} \delta_{\bar{x}_{i}}$ in the sense of measures.

The assumption $\operatorname{supp}\left(g_{0}\right)=\cup_{i=1}^{n} I_{i}^{\delta}$ can be relaxed to supp $g_{0}=\cup_{i=1}^{n} \tilde{I}_{i}$, where $\tilde{I}_{i}$ are small enough intervals containing $\bar{x}_{i}$, that is, those intervals need not be symetric around $\bar{x}_{i}$.

Our main results writes:

Theorem 3.9 Let $a, b$ satisfy Assumptions 1 and $\left(\bar{x}_{i}\right)_{i=1, \ldots, n}$ be an Evolutionary Attractor (in the sense of def 2.4). There exist $\lambda_{0}, \delta_{0}>0$ (depending on $\left.a, b,\left(\bar{x}_{i}\right)_{i=1, \ldots, n}\right)$, such that if $\lambda \in\left(0, \lambda_{0}\right), \delta \in\left(0, \delta_{0}\right), g_{0} \in L^{1}(X)$ satisfies Assumption 2 and $g$ is the solution of (3.9) given by Theorem 3.6. Then the asymptotic population distribution $f$ given by (3.11) is

$$
\forall t, \quad f(t, \cdot)=\sum_{i=1}^{n} \bar{\rho}_{i} \delta_{\bar{x}_{i}},
$$

where $\left(\bar{\rho}_{i}\right)_{i=1, \ldots, n}$ are defined by (2.6). 
Remark 3.10 Notice that in Theorem 3.9, the limit distribution $f$ of $f_{\varepsilon}$ is unique, thus the whole sequence $f_{\varepsilon}$ converges toward $f$ (and not only subsequences as in Theorem 3.6).

The hypothesis $\forall i=1, \ldots, n,\left|\int_{I_{i}^{\delta}} g-\bar{\rho}_{i}\right| \leq \lambda$ can be relaxed if a stronger assumption on the matrix $\left(b\left(\bar{x}_{i}, \bar{x}_{j}\right)\right)_{i, j=1, \ldots, n}$ is made.

One can find parameters $a$ and $b$ such that several Evolutionary Attractors exist. Numerically, we do observe, as expected, that each of them is locally stable (see subsection 5.2).

\section{Proof of Theorem 3.9.}

\subsection{A control on $\left(\int_{I_{i}^{\delta}} g(t, x) d x\right)_{i=1, \ldots, n}$.}

In the following proposition, we show that if Assumption 3 is satisfied for $\lambda, \delta$ small enough, then, $\left(\int_{I_{i}^{\delta}} g(t, x) d x\right)_{i=1, \ldots, n}$ remains close to $\left(\bar{\rho}_{i}\right)_{i=1, \ldots, n}$ at all times in the evolution of eq. (3.9).

Proposition 4.11 Let $a, b$ satisfy Assumption 1, and $\left(\bar{x}_{i}\right)_{i=1, \ldots, n}$ be a strategy. There exist $\lambda_{0}, \delta_{0}>0$ (depending on $\left.a, b,\left(\bar{x}_{i}\right)_{i=1, \ldots, n}\right)$, such that if $\lambda \in\left(0, \lambda_{0}\right), \delta \in\left(0, \delta_{0}\right), g_{0} \in L^{1}(X)$ satisfies Assumption 2, $g$ is the solution of (3.9) given by Theorem 3.6, and $\left(\bar{\rho}_{i}\right)_{i=1, \ldots, n}$ is defined in (2.6), then,

$$
\forall t \geq 0, \quad \forall i=1, \ldots, n, \quad\left|\int_{I_{i}^{\delta}} g(t, x) d x-\bar{\rho}_{i}\right| \leq \lambda .
$$

\section{Proof of Proposition 4.11.}

- Estimates on $\partial_{t} \int_{I_{i}^{\delta}} g(t, x) d x$ : for each $i \in\{1, \ldots, N\}$,

$$
\begin{aligned}
\forall x \in I_{i}^{\delta}, \quad \partial_{t} g_{\mid I_{i}^{\delta}}(t, x)= & \left(a(x)-\sum_{j=1}^{n} \int_{I_{j}^{\delta}} b(x, y) g(t, y) d y\right) g(t, x) \\
= & \left(a\left(\bar{x}_{i}\right)+O\left(x-\bar{x}_{i}\right)\right. \\
& \left.-\sum_{j=1}^{n} \int_{I_{j}^{\delta}}\left(b\left(\bar{x}_{i}, \bar{x}_{j}\right)+O\left(\left|x-\bar{x}_{i}\right|+\left|\bar{x}_{j}-y\right|\right)\right) g(t, y) d y\right) g(t, x) .
\end{aligned}
$$

Thus :

$$
\partial_{t} \int_{I_{i}^{\delta}} g(t, x) d x=\left(a\left(\bar{x}_{i}\right)-\sum_{j=1}^{n} b\left(\bar{x}_{i}, \bar{x}_{j}\right) \int_{I_{j}^{\delta}} g(t, x) d x\right) \int_{I_{i}^{\delta}} g(t, x) d x+O(\delta) .
$$

We already know thanks to (3.10) that $\|g\|_{L^{1}}$ is bounded. Then, we define $\tilde{\rho}_{i}(t):=$ $\int_{I_{i}^{\delta}} g(t, x) d x$, so that $\left(\tilde{\rho}_{i}\right)_{i=1, \ldots, n}$ satisfies:

$$
\forall i=1, \ldots, n, \quad \tilde{\rho}_{i}^{\prime}(t)=\left(a\left(\bar{x}_{i}\right)-\sum_{j=1}^{n} b\left(\bar{x}_{i}, \bar{x}_{j}\right) \tilde{\rho}_{j}(t)\right) \tilde{\rho}_{i}(t)+O(\delta) .
$$


From the definition of $\left(\bar{\rho}_{j}\right)_{j=1, \ldots, n}$, we know that $a\left(\bar{x}_{i}\right)=\sum_{j=1}^{n} b\left(\bar{x}_{i}, \bar{x}_{j}\right) \bar{\rho}_{j}$, so that:

$$
\begin{array}{r}
\forall i=1, \ldots, n, \quad \tilde{\rho}_{i}^{\prime}(t)=-\left(\sum_{j=1}^{n} b\left(\bar{x}_{i}, \bar{x}_{j}\right)\left(\tilde{\rho}_{j}(t)-\bar{\rho}_{j}\right)\right) \tilde{\rho}_{i}(t)+O(\delta), \quad(4.14) \\
\forall i=1, \ldots, n, \quad\left(\tilde{\rho}_{i}-\bar{\rho}_{i}\right)^{\prime}(t)=-\left(\sum_{j=1}^{n} b\left(\bar{x}_{i}, \bar{x}_{j}\right)\left(\tilde{\rho}_{j}(t)-\bar{\rho}_{j}\right)\right) \bar{\rho}_{i}+O(\delta)+O\left(\left\|\tilde{\rho}_{i}-\bar{\rho}_{i}\right\|_{\infty}^{2}\right),
\end{array}
$$

- We can write eq. (4.15) as follow:

$$
\forall i=1, \ldots, n, \quad\left(\tilde{\rho}_{i}-\bar{\rho}_{i}\right)^{\prime}(t)=\left(D F_{\left(\bar{\rho}_{i}\right)_{i=1, \ldots, n}}(\tilde{\rho}(t)-\bar{\rho})\right)_{i}+O(\delta)+O\left(\|\tilde{\rho}-\bar{\rho}\|_{\infty}^{2}\right),
$$

where $D F_{\left(\bar{\rho}_{i}\right)_{i=1, \ldots, n}}=-\operatorname{diag}\left(\left(\bar{\rho}_{i}\right)_{i=1, \ldots, n}\right)\left(b\left(\bar{x}_{i}, \bar{x}_{j}\right)\right)_{i, j}$ (see def 2.1). The solution of this system of ordinary differential equations is given by:

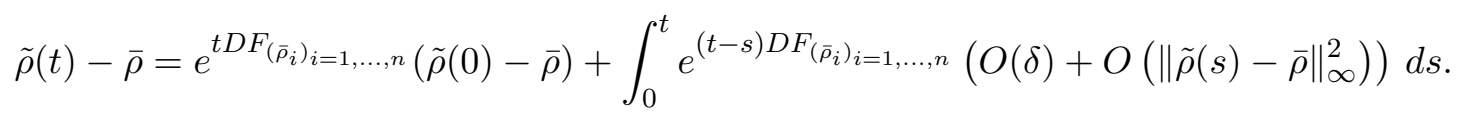

Thus:

$\|\tilde{\rho}(t)-\bar{\rho}\|_{\infty} \leq\left\|e^{t D F_{\left(\bar{\rho}_{i}\right)_{i=1, \ldots, n}}}\right\|_{\infty}\|\tilde{\rho}(0)-\bar{\rho}\|_{\infty}+\int_{0}^{t}\left\|e^{(t-s) D F_{\left(\bar{\rho}_{i}\right)_{i=1, \ldots, n}}}\right\|_{\infty}\left(O(\delta)+O\left(\|\tilde{\rho}(s)-\bar{\rho}\|_{\infty}^{2}\right)\right) d s$

As $\left(\bar{x}_{i}\right)_{i}$ is a strategy (see def 2.1), the eigenvalues of the matrix $D F_{\left(\bar{\rho}_{i}\right)_{i=1, \ldots, n}}$ have a negative real part: $\left.\mathcal{R} e\left[\operatorname{Spec}\left(D F_{\left(\bar{\rho}_{i}\right)_{i=1, \ldots, n}}\right)\right] \subset\right]-\infty,-\mu[, \mu>0$. This provides the estimate $\left\|e^{t D F_{\left(\bar{\rho}_{i}\right)_{i=1, \ldots, n}}}\right\|_{\infty} \leq O\left(t^{n}\right) e^{-t \mu}$, and then:

$$
\begin{aligned}
\|\tilde{\rho}(t)-\bar{\rho}\|_{\infty} & \leq e^{-t \mu} O\left(t^{n}\right)\|\tilde{\rho}(0)-\bar{\rho}\|_{\infty}+\int_{0}^{t} O\left((t-s)^{n}\right) e^{-(t-s) \mu}\left(O(\delta)+O\left(\|\tilde{\rho}(s)-\bar{\rho}\|_{\infty}^{2}\right)\right) d s \\
& \leq C_{1}\|\tilde{\rho}(0)-\bar{\rho}\|_{\infty}+\left(O(\delta)+\sup _{s \in[0, t)}\|\tilde{\rho}(s)-\bar{\rho}\|_{\infty}^{2} \int_{0}^{t} O\left((t-s)^{n}\right) e^{-(t-s) \mu} d s\right) \\
& \leq C_{1}\|\tilde{\rho}(0)-\bar{\rho}\|_{\infty}+O(\delta)+C_{2}\left(\sup _{s \in[0, t)}\|\tilde{\rho}(s)-\bar{\rho}\|_{\infty}\right)^{2} .
\end{aligned}
$$

We assume that $\lambda<\frac{1}{4 C_{2}},\|\tilde{\rho}(0)-\bar{\rho}\|_{\infty}<\frac{\lambda}{4 C_{1}}<\lambda$, and $\delta>0$ is small enough (so that $\left.O(\delta)<\frac{1}{4}\right)$. Let define $T:=\sup \left\{T_{1}>0 ; \forall t<T_{1},\|\tilde{\rho}(t)-\bar{\rho}\|_{\infty}<\lambda\right\} \in[0, \infty]$. As $\tilde{\rho}(\cdot)-\bar{\rho}$ is continuous and $\left\|\left(\tilde{\rho}_{i}-\bar{\rho}_{i}\right)(0)\right\|_{\infty}<\lambda$, we see that $T>0$. If $T<\infty$, then: 


$$
\begin{aligned}
\|\tilde{\rho}(T)-\bar{\rho}\|_{\infty} & \leq C_{1} \frac{\lambda}{4 C_{1}}+\frac{\lambda}{4}+C_{2}\left(\frac{1}{4 C_{2}}\right) \lambda \\
& \leq \frac{3}{4} \lambda .
\end{aligned}
$$

Thus, $T=\infty$, which proves (4.13).

From now on, we define for $f$ given in Theorem 3.6 (note that $\mathrm{f}$ can be a measure with respect to $x)$ :

$$
\rho_{i}(t):=\int_{I_{i}^{\delta}} f(t, d x) .
$$

Thanks to Prop. 4.11, it is possible to show that $\left(\rho_{i}(t)\right)_{i=1, \ldots, n}$ is close to $\left(\bar{\rho}_{i}\right)_{i=1, \ldots, n}$ :

Corollary 4.12 Let $a, b$ satisfy Assumption 1 , and $\left(\bar{x}_{i}\right)_{i=1, \ldots, n}$ a strategy. There exist $\lambda_{0}, \delta_{0}>$ 0 (depending on $\left.a, b,\left(\bar{x}_{i}\right)_{i=1, \ldots, n}\right)$, such that if $\lambda \in\left(0, \lambda_{0}\right), \delta \in\left(0, \delta_{0}\right), g_{0} \in L^{1}(X)$ satisfies Assumption 2, $g$ is the solution of (3.9) given by Theorem 3.6, and $\left(\bar{\rho}_{i}\right)_{i=1, \ldots, n}$ defined by (2.6), then the asymptotic population distribution $f$ given by (3.11) in Thm 3.6 has its support in $\cup_{i=1, \ldots, n} I_{i}^{\delta}$, and:

$$
\forall i=1, \ldots, n, \quad\left|\rho_{i}(t)-\bar{\rho}_{i}\right| \leq \lambda \text { for a.e. } t \in \mathbb{R}_{+} .
$$

Proof of Corollary 4.12. Let $\varphi \in C_{b}(\mathbb{R})$, and $0<T<T^{\prime}$,

$$
\begin{aligned}
\int_{\mathbb{R}_{+}} \int_{X} \varphi(x) \chi_{\left[T, T^{\prime}\right]} d f(t)(x) & =\lim _{m \rightarrow \infty} \int_{\mathbb{R}_{+}} \int_{X} \varphi(x) \chi_{\left[T, T^{\prime}\right]} d f_{\varepsilon_{m}}(t)(x) \\
& =\lim _{m \rightarrow \infty} \int_{T}^{T^{\prime}} \int_{X} g\left(\frac{t}{\varepsilon_{m}}, x\right) \varphi(x) d x d t
\end{aligned}
$$

and since $\operatorname{supp} g(t, \cdot) \subset \cup_{i=1}^{n} I_{i}^{\delta}$,

$$
\int_{\mathbb{R}_{+}} \int_{X} \varphi(x) \chi_{\left[T, T^{\prime}\right]} d f(t)(x)=\lim _{m \rightarrow \infty} \sum_{i=1}^{n} \int_{T}^{T^{\prime}} \int_{I_{i}^{\delta}} g\left(\frac{t}{\varepsilon_{m}}, x\right) \varphi(x) d x d t .
$$

Thus, if $\operatorname{supp}(\varphi) \subset\left(\cup_{i=1, \ldots, n} I_{i}^{\delta}\right)^{c}$, then $\iint \varphi d f=0$, that is $\operatorname{supp}(f(t, \cdot)) \subset \cup_{i=1, \ldots, n} I_{i}^{\delta}$.

Let $i \in\{1, \ldots, n\}$. If $\varphi$ is a cut-off $C_{b}(\mathbb{R})$ function such that $\left.\varphi\right|_{I_{i}^{\delta}} \equiv 1$ and $\left.\varphi\right|_{\cup_{j \neq i} I_{j}^{\delta}} \equiv 0$, then,

$$
\int_{T}^{T^{\prime}} \int_{X} \varphi d f=\lim _{m \rightarrow \infty} \int_{T}^{T^{\prime}} \int_{I_{i}^{\delta}} g\left(\frac{t}{\varepsilon_{m}}, x\right) d x d t .
$$

Thanks to (4.13), for $n$ large enough,

$$
\left|\int_{I_{i}^{\delta}} g\left(\frac{t}{\varepsilon_{m}}, x\right) d x-\bar{\rho}_{i}\right|<\lambda .
$$


Thus:

$$
\left|\int_{T}^{T^{\prime}}\left(\int_{I_{i}^{\delta}} g\left(\frac{t}{\varepsilon_{m}}, x\right) d x-\bar{\rho}_{i}\right) \frac{d t}{\left|T^{\prime}-T\right|}\right|<\lambda .
$$

Since this is true for all $0<T<T^{\prime}$, the following inequality is true almost everywhere in time:

$$
\left|\int_{X} \varphi(x) d f(t)(x)-\bar{\rho}_{i}\right|<\lambda .
$$

Since $\operatorname{supp}(f) \subset \cup_{i=1, \ldots, n} I_{i}^{\delta}$, we get the result.

\section{2 $f$ is a sum of Dirac masses.}

In this subsection, we first prove that $f$ is a sum of Dirac masses $f(t, x)=\sum_{i=1}^{n} \rho_{i}(t) \delta_{x_{i}(t)}$.

Then, and that's the difficult part of this subsection, we show that $t \mapsto\left(x_{i}(t)\right)_{i=1, \ldots, n}$ is smooth. This requires several steps: We first show the Hölder and Lipschitz regularity of $\left(x_{i}(\cdot)\right)_{i=1, \ldots, n}$, and then we prove an equality which provides the Lipschitz regularity of $\left(\rho_{i}(\cdot)\right)_{i=1, \ldots, n}$. Thanks to the information about the smoothness of $\left(x_{i}(\cdot)\right)_{i=1, \ldots, n}$, we are then able to write down a differential equation satisfied by $\left(x_{i}(\cdot)\right)_{i=1, \ldots, n}$.

Proposition 4.13 Let a, $b$ satisfy Assumption 1, and $\left(\bar{x}_{i}\right)_{i=1, \ldots, n}$ be an ESS. There exist $\lambda_{0}, \delta_{0}>$ 0 (depending on $\left.a, b,\left(\bar{x}_{i}\right)_{i=1, \ldots, n}\right)$, such that if $\lambda \in\left(0, \lambda_{0}\right), \delta \in\left(0, \delta_{0}\right), g_{0} \in L^{1}(X)$ satisfies Assumption 2, $g$ is the solution of (3.9) given by Theorem 3.6, $f$ is the asymptotic population distribution given by (3.11), and $\left(\bar{\rho}_{i}\right)_{i=1, \ldots, n},\left(\rho_{i}(\cdot)\right)_{i=1, \ldots, n}$ are defined in (2.6), (4.16), then there exists $C>0$ such that $R$ (defined in (3.12) in Thm 3.6) satisfies:

$$
\forall x \in I^{\delta}, \quad \forall t \geq 0, \quad \partial_{x x}^{2} R(t, x)<-C t,
$$

and $f$ can be written as:

$$
f(t, x)=\sum_{i=1}^{n} \rho_{i}(t) \delta_{x_{i}(t)}(x), \text { for a.e. } t>0
$$

where $x_{i}(t) \in I_{i}^{\delta}, \forall i=1, \ldots, n$.

\section{Proof of Proposition 4.13.}

Thanks to Corollary 4.12, we know that for all $i=1, \ldots, n, \operatorname{supp}(f(t, \cdot)) \cap I_{i}^{\delta} \neq \emptyset$. Thus, thanks to Proposition 3.6, for each $i=1, \ldots, n$, there exists $x_{i}(t) \in I_{i}^{\delta}$ such that $R\left(t, x_{i}(t)\right)=0$. We also know from Proposition 3.6 that for all $x \in I_{i}^{\delta}, \quad R(t, x) \leq 0$. In order to prove the uniqueness of $x_{i}(t)$, it is sufficient to show that $\partial_{x x}^{2} R(t, \cdot)_{\mid I^{\delta}}<0$. 
Let $i \in\{1, \ldots, n\}$, and $x \in I_{i}^{\delta}$.

$$
\begin{aligned}
\partial_{x x}^{2} R(t, \cdot)(x)= & \int_{0}^{t}\left[a^{\prime \prime}(x)-\int_{X} \partial_{x x}^{2} b(x, y) f(\sigma, y) d y\right] d \sigma \\
= & \int_{0}^{t}\left(a^{\prime \prime}(x)-\sum_{j} \int_{I_{j}^{\delta}} \partial_{x x}^{2} b(x, y) d(f(\sigma))(y)\right) d \sigma \\
= & \int_{0}^{t}\left[a^{\prime \prime}\left(\bar{x}_{i}\right)+\left(a^{\prime \prime}(x)-a^{\prime \prime}\left(\bar{x}_{i}\right)\right)-\sum_{j=1}^{n}\left(\partial_{x x}^{2} b\left(\bar{x}_{i}, \bar{x}_{j}\right)\right) \bar{\rho}_{j}\right. \\
& -\sum_{j=1}^{n}\left(\int_{I_{j}^{\delta}} \partial_{x x}^{2} b(x, y) d(f(\sigma))(y)-\int_{I_{j}^{\delta}} \partial_{x x}^{2} b(x, y) \frac{\bar{\rho}_{j}}{\left|I_{j}^{\delta}\right|} d y\right. \\
& \left.\left.-\int_{I_{j}^{\delta}}\left(\partial_{x x}^{2} b(x, y)-\partial_{x x}^{2} b\left(\bar{x}_{i}, \bar{x}_{j}\right)\right) \frac{\bar{\rho}_{j}}{\left|I_{j}^{\delta}\right|} d y\right)\right] d \sigma \\
\leq & \int_{0}^{t}\left[\partial_{x x}^{2} s_{\sum_{i=1}^{n} \bar{\rho}_{i} \delta_{\bar{x}_{j}}}\left(\bar{x}_{i}\right)+o_{\delta}(1)+n\left\|\partial_{x x}^{2} b\right\|_{\infty} \lambda\right] d \sigma
\end{aligned}
$$

thanks to Corollary 4.12. Since $\left(\bar{x}_{i}\right)_{i=1, \ldots, n}$ is an ESS, one has $\forall j=1, \ldots, n, \partial_{x x}^{2} s_{\sum_{k=1}^{n} \bar{\rho}_{k} \delta_{\bar{x}_{k}}}\left(\bar{x}_{j}\right)<$ 0 . Thus, if $\lambda$ is small enough,

$$
\partial_{x x}^{2} R(t, \cdot)_{\mid I_{i}^{\delta}}<C t<0
$$

Finally, $f$ can be written under the form $f(t, x)=\sum_{i=1}^{n} r_{i}(t) \delta_{x_{i}(t)}$, where $\forall i=1, \ldots, n, x_{i}(t) \in$ $I_{i}^{\delta}$, and thus $\forall i=1, \ldots, n, r_{i}(t)=\int_{I_{i}^{\delta}} f=\rho_{i}(t)$.

Remark 4.14 Thanks to Remark 3.7, if $x_{i}(t) \notin \partial I_{i}^{\delta}$, then $\partial_{x} R\left(t, x_{i}(t)\right)=0$. If $x_{i}(t)=\bar{x}_{i}-\delta$ (resp. $\left.x_{i}(t)=\bar{x}_{i}+\delta\right)$, then $\partial_{x} R\left(t, x_{i}(t)\right) \leq 0$ (resp. $\left.\partial_{x} R\left(t, x_{i}(t)\right) \geq 0\right)$. As a consequence, if $x_{i}(t) \in \partial I_{i}^{\delta}$,

$\forall x \in I_{i}^{\delta}, \operatorname{sgn}\left(\partial_{x} R\left(t, x_{i}(t)\right)\right)=\operatorname{sgn}\left(x_{i}(t)-\bar{x}_{i}\right)=\operatorname{sgn}\left(x_{i}(t)-x\right)$.

Proposition 4.15 Let $a, b$ satisfy Assumption 1, and $\left(\bar{x}_{i}\right)_{i=1, \ldots, n}$ be an ESS. There exist $\lambda_{0}, \delta_{0}>$ 0 (depending on $\left.a, b,\left(\bar{x}_{i}\right)_{i=1, \ldots, n}\right)$, such that if $\lambda \in\left(0, \lambda_{0}\right), \delta \in\left(0, \delta_{0}\right), g_{0} \in L^{1}(X)$ satisfies Assumption 2, $g$ is the solution of (3.9) given by Theorem 3.6, $f$ is the asymptotic population distribution given by (3.11), and $\left(\bar{\rho}_{i}\right)_{i=1, \ldots, n},\left(\rho_{i}(\cdot)\right)_{i=1, \ldots, n},\left(x_{i}(\cdot)\right)_{i=1, \ldots, n}$ are defined in (2.6), (4.16), (4.19), then,

1. $\forall i=1, \ldots, n, x_{i}(\cdot)$ is Hölder $\frac{1}{2}$ on $[T, \infty)$ a.e., for all $T>0$, that is

$$
\exists C>0 \text {, for a.e. } t>T, \text { for a.e. }|h| \leq \frac{T}{2}, \quad\left|x_{i}(t)-x_{i}(t+h)\right| \leq C|h|^{\frac{1}{2}} .
$$


2. $\forall i=1, \ldots, n, x_{i}(\cdot)$ is Lipschitz continuous a.e. on $[T, \infty) \cap\left\{t ; x_{i}(t) \in \operatorname{Int}\left(I_{i}^{\delta}\right)\right\}$ for all $T>0$, that is

$$
\exists C>0, \text { for a.e. } t, t^{\prime} \in \subset[T, \infty) \cap\left\{t ; x_{i}(t) \in \operatorname{Int}\left(I_{i}^{\delta}\right)\right\}, \quad\left|x_{i}(t)-x_{i}\left(t^{\prime}\right)\right| \leq C\left|t-t^{\prime}\right| .
$$

3. For a.e. $t \in \mathbb{R}_{+} \cap\left\{t ; x_{i}(t) \in \operatorname{Int}\left(I_{i}^{\delta}\right)\right\}, x_{i}$ and $\rho_{i}$ satisfy the equation

$$
0=a\left(x_{i}(t)\right)-\sum_{j=1}^{n} b\left(x_{j}(t), x_{i}(t)\right) \rho_{j}(t) .
$$

4. $\forall i=1, \ldots, n, \rho_{i}(\cdot)$ is Lipschitz continuous a.e. on $[T, \infty) \cap\left\{t ; x_{i}(t) \in \operatorname{Int}\left(I_{i}^{\delta}\right)\right\}$ for all $T>0$, that is

$$
\exists C>0, \text { for a.e. } t, t^{\prime} \in \subset[T, \infty) \cap\left\{t ; x_{i}(t) \in \operatorname{Int}\left(I_{i}^{\delta}\right)\right\} \text { a.e., } \quad\left|\rho_{i}(t)-\rho_{i}\left(t^{\prime}\right)\right| \leq C\left|t-t^{\prime}\right| .
$$

\section{Proof of Proposition 4.15.}

1. We use here the parabola-like shape of $x \mapsto R(t, x)$. Let $i \in\{1, \ldots, n\}$, then

$$
\begin{aligned}
R\left(t+h, x_{i}(t)\right) & =R\left(t, x_{i}(t)\right)+h a\left(x_{i}(t)\right)-\int_{t}^{t+h} \sum_{j=1}^{n} b\left(x_{i}(t), x_{j}(\sigma)\right) \rho_{j}(\sigma) d \sigma \\
\geq & h a\left(x_{i}(t)\right)-h\|b\|_{\infty} \sup _{\sigma \in \mathbb{R}}\|f(\sigma, \cdot)\|_{M^{1}} \\
\geq-C h . & \\
R\left(t+h, x_{i}(t)\right)= & R\left(t+h, x_{i}(t+h)\right)+\left(x_{i}(t)-x_{i}(t+h)\right) \partial_{x} R\left(t+h, x_{i}(t+h)\right) \\
& +\frac{1}{2}\left(x_{i}(t)-x_{i}(t+h)\right)^{2} \partial_{x x}^{2} R(t+h, \theta) \text { for some } \theta \in\left[x_{i}(t), x_{i}(t+h)\right] \\
\leq & -\frac{C(t-|h|)}{2}\left(x_{i}(t)-x_{i}(t+h)\right)^{2},
\end{aligned}
$$

because if $x_{i}(t+h) \in \operatorname{Int}\left(I_{i}^{\delta}\right)$, then $\partial_{x} R\left(t+h, x_{i}(t+h)\right)=0$, and if $x_{i}(t+h) \in \partial I_{i}^{\delta}$, then $\operatorname{sgn}\left(\partial_{x} R\left(t+h, x_{i}(t+h)\right)\right)=-\operatorname{sgn}\left(x_{i}(t)-x_{i}(t+h)\right)$ thanks to Remark 4.14. We get the regularity result:

$$
\left|x_{i}(t)-x_{i}(t+h)\right| \leq C\left(\frac{|h|}{t}\right)^{\frac{1}{2}}+O(|h|) .
$$

Notice that thank to this estimate, for each $i=1, \ldots, n,\left\{t \geq 0 ; x_{i}(t) \in \operatorname{Int}\left(I_{i}^{\delta}\right)\right\}$ is an open set of $\mathbb{R}_{+}$.

2. Let $i \in\{1, \ldots, n\}$, and $t \in\left\{t \geq 0 ; x_{i}(t) \in \operatorname{Int}\left(I_{i}^{\delta}\right)\right\}$. This set is open in $\mathbb{R}_{+}$and $x_{i}(\cdot)$ is continuous, thus, if $h$ is small enough, $x_{i}(t+h) \in \operatorname{Int}\left(I_{i}^{\delta}\right)$. Thanks to Remark 4.14, $0=\partial_{x} R\left(t+h, x_{i}(t+h)\right)=\partial_{x} R\left(t, x_{i}(t)\right)$. Then: 


$$
\begin{aligned}
0= & \partial_{x} R\left(t+h, x_{i}(t+h)\right)-\partial_{x} R\left(t, x_{i}(t)\right) \\
= & h\left(a^{\prime}\left(x_{i}(t+h)\right)-\int_{t}^{t+h} \sum_{j=1}^{n} \partial_{x} b\left(x_{i}(t+h), x_{j}(\sigma)\right) \rho_{j}(\sigma) \frac{d \sigma}{h}\right) \\
& +\partial_{x} R\left(t, x_{i}(t+h)\right)-\partial_{x} R\left(t, x_{i}(t)\right) .
\end{aligned}
$$

We can estimate the two terms of this equality:

$$
\begin{aligned}
& \left|h\left(a^{\prime}\left(x_{i}(t+h)\right)-\int_{t}^{t+h} \sum_{j=1}^{n} \partial_{x} b\left(x_{i}(t+h), x_{j}(\sigma)\right) \rho_{j}(\sigma) \frac{d \sigma}{h}\right)\right| \leq\left(\left\|a^{\prime}\right\|_{\infty}+\left\|\partial_{x} b\right\|_{\infty}\|f\|_{L^{\infty}\left(L^{1}\right)}\right)|h|, \\
& \partial_{x} R\left(t, x_{i}(t+h)\right)-\partial_{x} R\left(t, x_{i}(t)\right)=\left(x_{i}(t+h)-x_{i}(t)\right) \partial_{x x}^{2} R\left(t, x_{i}(t)\right)+O\left(t\left(x_{i}(t+h)-x_{i}(t)\right)^{2}\right) .
\end{aligned}
$$

Thus, identity (4.20) provides the following equality:

$$
0=O(h)+\left(x_{i}(t+h)-x_{i}(t)\right) \partial_{x x}^{2} R\left(t, x_{i}(t)\right)+O\left(t\left(x_{i}(t+h)-x_{i}(t)\right)^{2}\right) .
$$

Thanks to part 1 of this proposition, $\left(x_{i}(t+h)-x_{i}(t)\right)^{2}=\frac{1}{t} O(|h|)$, thus:

$$
\left(x_{i}(t+h)-x_{i}(t)\right) \partial_{x x}^{2} R\left(t, x_{i}(t)\right)=O(|h|) .
$$

Thanks to Proposition 4.13, $\partial_{x x}^{2} R\left(t, x_{i}(t)\right)<-C t<0$, thus $x_{i}(\cdot)$ is Lipschitz continuous on $[T, \infty)$ for all $T>0$ :

$$
\left|x_{i}(t+h)-x_{i}(t)\right|<\frac{\tilde{C}|h|}{t}=\frac{1}{t} O(|h|) .
$$

3. Let $i \in\{1, \ldots, n\}$, then

$$
\begin{aligned}
0= & R\left(t+h, x_{i}(t+h)\right)-R\left(t, x_{i}(t)\right) \\
= & h\left(a\left(x_{i}(t+h)\right)-\int_{t}^{t+h} \sum_{j=1}^{n} b\left(x_{i}(t+h), x_{j}(\sigma)\right) \rho_{j}(\sigma) \frac{d \sigma}{h}\right) \\
& +R\left(t, x_{i}(t+h)\right)-R\left(t, x_{i}(t)\right) .
\end{aligned}
$$

But $\rho_{j} \in L^{\infty}\left(\mathbb{R}_{+}\right)$, thus almost every $t$ is a Lebesgue point (see [13]). As a consequence, for a.e. $t>0$, as $h \rightarrow 0$,

$$
\begin{aligned}
\int_{t}^{t+h} \sum_{j=1}^{n} b\left(x_{i}(t+h), x_{j}(\sigma)\right) \rho_{j}(\sigma) \frac{d \sigma}{h}= & \int_{t}^{t+h} \sum_{j=1}^{n} b\left(x_{i}(t), x_{j}(\sigma)\right) \rho_{j}(\sigma) \frac{d \sigma}{h} \\
& +O_{\tilde{C},\left\|\partial_{x} b\right\|_{\infty}(h)} \\
\rightarrow & \sum_{j=1}^{n} b\left(x_{i}(t), x_{j}(t)\right) \rho_{j}(t) .
\end{aligned}
$$


We can also estimate the other terms of the above equation:

$$
\begin{aligned}
a\left(x_{i}(t+h)\right)= & a\left(x_{i}(t)\right)+O\left(x_{i}(t+h)-x_{i}(t)\right), \\
R\left(t, x_{i}(t+h)\right)-R\left(t, x_{i}(t)\right)= & \left(x_{i}(t+h)-x_{i}(t)\right) \partial_{x} R\left(t, x_{i}(t)\right) \\
& +O\left(t\left(x_{i}(t+h)-x_{i}(t)\right)^{2}\right) \\
= & O\left(t\left(x_{i}(t+h)-x_{i}(t)\right)^{2}\right) \\
= & O\left(t|h|^{2}\right),
\end{aligned}
$$

when $t \in\left\{t \geq 0 ; x_{i}(t) \in \operatorname{Int}\left(I_{i}^{\delta}\right)\right\}$ (since $0=\partial_{x} R\left(t, x_{i}(t)\right)$, thanks to Remark 4.14).

Let $t \in\left\{t \geq 0 ; x_{i}(t) \in \operatorname{Int}\left(I_{i}^{\delta}\right)\right\}$ such that (4.24) holds (this is true a.e.). We shall show the result for this particular $t$. Identity (4.23) provides the following equality (here, $O$ and $o$ may depend on $t$ ):

$$
0=h\left(a\left(x_{i}(t)\right)+O\left(x_{i}(t+h)-x_{i}(t)\right)-\sum_{j=1}^{n} b\left(x_{i}(t), x_{j}(t)\right) \rho_{j}(t)+o_{h}(1)\right)+O\left(|h|^{2}\right) .
$$

But $x_{i}(t+h)-x_{i}(t)=O\left(|h|^{\frac{1}{2}}\right)$, thus, if we divide (4.26) by $h$, we get:

$$
a\left(x_{i}(t)\right)-\sum_{j=1}^{n} b\left(x_{i}(t), x_{j}(t)\right) \rho_{j}(t)=O(|h|)+o_{h}(1) \rightarrow_{h \rightarrow 0} 0,
$$

that is $a\left(x_{i}(t)\right)-\sum_{j=1}^{n} b\left(x_{i}(t), x_{j}(t)\right) \rho_{j}(t)=0$. Since this is true for a.e. $t \in\left\{t \geq 0 ; x_{i}(t) \in\right.$ $\left.\operatorname{Int}\left(I_{i}^{\delta}\right)\right\}$, we get the result.

4. Thanks to Assumption 2, the matrix $\left(b\left(\bar{x}_{i}, \bar{x}_{j}\right)\right)_{i, j=1, \ldots, n}$ is invertible, and so is also $\left(b\left(x_{i}(t), x_{j}(t)\right)\right)_{i, j=1, \ldots, n}$ if $\delta$ is small enough. The result follows from the cofactor formula.

Thanks to the regularity of $\left(x_{i}(\cdot)\right)_{i=1, \ldots, n},\left(\rho_{i}(\cdot)\right)_{i=1, \ldots, n}$, we can define those functions for all $t>0$ (and not only for a.e. $t \geq 0$ ).

Proposition 4.16 Let $a, b$ satisfy Assumption 1, and $\left(\bar{x}_{i}\right)_{i=1, \ldots, n}$ be an ESS. There exist $\lambda_{0}, \delta_{0}>$ 0 (depending on $\left.a, b,\left(\bar{x}_{i}\right)_{i=1, \ldots, n}\right)$, such that if $\lambda \in\left(0, \lambda_{0}\right), \delta \in\left(0, \delta_{0}\right), g_{0} \in L^{1}(X)$ satisfies Assumption 3, $g$ is the solution of (3.9) given by Theorem 3.6, $f$ is the asymptotic population distribution given by (3.11), and $\left(\bar{\rho}_{i}\right)_{i=1, \ldots, n},\left(\rho_{i}(\cdot)\right)_{i=1, \ldots, n},\left(x_{i}(\cdot)\right)_{i=1, \ldots, n}$ are defined in (2.6), (4.16), (4.19), then, for each $i=1, \ldots, n, x_{i}(\cdot)$ is $C^{1}$ on $[T, \infty) \cap\left\{t ; x_{i}(t) \in \operatorname{Int}\left(I_{i}^{\delta}\right)\right\}$ for all $T>0$, and:

$$
x_{i}^{\prime}(t)=\frac{\partial_{x} s_{\sum_{j=1}^{n} \rho_{j}(t) \delta_{x_{j}(t)}}\left(x_{i}(t)\right)}{-\partial_{x x}^{2} R\left(t, x_{i}(t)\right)} .
$$


Proof of Proposition 4.16. We proceed as in the proof of part 2 of Proposition 4.15. Formula (4.21) still holds, and thanks to the regularity of $\left(x_{i}(\cdot)\right)_{i=1, \ldots, n},\left(\rho_{i}(\cdot)\right)_{i=1, \ldots, n}$ (see parts 2 and 4 of Proposition 4.15),

$$
a^{\prime}\left(x_{i}(t+h)\right)-\int_{t}^{t+h} \sum_{j=1}^{n} \partial_{x} b\left(x_{i}(t+h), x_{j}(\sigma)\right) \rho_{j}(\sigma) \frac{d \sigma}{h} \rightarrow \partial_{x} s_{\sum_{j=1}^{n} \rho_{j}(t) \delta_{x_{j}(t)}}\left(x_{i}(t)\right) .
$$

Using this estimate, identity (4.20) becomes:

$$
\begin{aligned}
0= & h \partial_{x} s_{\sum_{j=1}^{n} \rho_{j}(t) \delta_{x_{j}(t)}}\left(x_{i}(t)\right)+o(h) \\
& +\left(x_{i}(t+h)-x_{i}(t)\right) \partial_{x x}^{2} R\left(t, x_{i}(t)\right)+O\left(\left(x_{i}(t+h)-x_{i}(t)\right)^{2}\right) .
\end{aligned}
$$

We know from part 2 of Proposition 4.15, that $\left(x_{i}(t)\right)_{i=1, \ldots, n}$ is Lipschitz-continuous, thus

$$
0=h \partial_{x} s_{\sum_{j=1}^{n} \rho_{j}(t) \delta_{x_{j}(t)}}\left(x_{i}(t)\right)+\left(x_{i}(t+h)-x_{i}(t)\right) \partial_{x x}^{2} R\left(t, x_{i}(t)\right)+o(h) .
$$

From Proposition 4.13, we know that $\partial_{x x}^{2} R\left(t, x_{i}(t)\right)<-C t<0$, wich provides the result.

We now prove the following technical lemma:

Lemma 4.1 If $I$ is an interval of $\mathbb{R}, J$ an open set of $I, K>0, c \in \mathbb{R}$, and $h: I \mapsto \mathbb{R}$ is such that:

$$
\left\{\begin{array}{l}
h \text { is continuous on } I, \\
h \text { is } K \text {-Lipschitz continuous on each connected component of } J: \\
\quad \forall[x, y] \subset J,\|h(x)-h(y)\| \leq K\|x-y\|, \\
h=c \text { on } I \backslash J,
\end{array}\right.
$$

then $h$ is K-Lipschitz continuous on I.

Proof of Lemma 4.1. Let $x, y \in I, x<y$. We want to prove that:

$$
|h(x)-h(y)| \leq K|x-y| .
$$

If $(x, y) \subset J$, this follows from the $K$-lipschitz continuity of $h$ on $J$. Otherwise, we define:

$$
\begin{aligned}
& \bar{x}=\inf \{\tilde{x}>x ; \tilde{x} \notin J\}, \\
& \bar{y}=\sup \{\tilde{y}<y ; \tilde{y} \notin J\} .
\end{aligned}
$$

Since $h$ is continuous, $h(\bar{x})=h(\bar{y})=c$. If $x \neq \bar{x}$ (resp. $y \neq \bar{y}$ ), then $h$ is $K$-Lipschitz continuous on $(x, \bar{x})$ (resp. $(\bar{y}, y))$, and thus:

$$
|h(x)-h(\bar{x})| \leq K|x-\bar{x}|, \quad(\text { resp. }|h(y)-h(\bar{y})| \leq K|y-\bar{y}|) .
$$


Then,

$$
\begin{aligned}
|h(x)-h(y)| & \leq|h(x)-h(\bar{x})|+|h(\bar{x})-h(\bar{y})|+|h(\bar{y})-h(y)|, \\
& \leq K|x-\bar{x}|+0+K|y-\bar{y}|, \\
& \leq K|x-y| .
\end{aligned}
$$

We get thus inequality (4.27). Since this is true for all $x, y \in I$, we get the $K$-Lipschitz continuity of $h$.

Proposition 4.17 Let a, $b$ satisfy Assumptions 1, and $\left(\bar{x}_{i}\right)_{i=1, \ldots, n}$ be an ESS. There exist $\lambda_{0}, \delta_{0}>$ 0 (depending on $\left.a, b,\left(\bar{x}_{i}\right)_{i=1, \ldots, n}\right)$, such that if $\lambda \in\left(0, \lambda_{0}\right), \delta \in\left(0, \delta_{0}\right), g_{0} \in L^{1}(X)$ satisfies Assumption 2, $g$ is the solution of (3.9) given by Theorem 3.6, $f$ is the asymptotic population distribution given by (3.11), and $\left(\bar{\rho}_{i}\right)_{i=1, \ldots, n},\left(\rho_{i}(\cdot)\right)_{i=1, \ldots, n},\left(x_{i}(\cdot)\right)_{i=1, \ldots, n}$ are defined in (2.6), (4.16), (4.19), then

1. $\left(x_{i}(\cdot)\right)_{i=1, \ldots, n}$ is uniformly Lipschitz continuous on $[R,+\infty)$, for all $R>0$ :

$$
\forall R>0, \exists K>0, \forall t, t^{\prime} \geq R,\left|x_{i}(t)-x_{i}\left(t^{\prime}\right)\right| \leq K\left|t-t^{\prime}\right|
$$

2. $\forall t>0$,

$$
0=a\left(x_{i}(t)\right)-\sum_{j=1}^{n} b\left(x_{j}(t), x_{i}(t)\right) \rho_{j}(t)
$$

\section{Proof of Proposition 4.17.}

1. Let $i \in\{1, \ldots, n\}$. We know from Prop.4.16 that:

$$
\forall t \in\left\{t>0 ; x_{i}(t) \in \operatorname{Int}\left(I_{i}^{\delta}\right)\right\}, \quad x_{i}^{\prime}(t)=\frac{\partial_{x} s_{\sum_{j=1}^{n} \rho_{j}(t) \delta_{x_{j}(t)}}\left(x_{i}(t)\right)}{-\partial_{x x}^{2} R\left(t, x_{i}(t)\right)} .
$$

From Proposition 4.13, we know that $\left|\partial_{x x}^{2} R\left(t, x_{i}(t)\right)\right|>C t$, thus,

$$
\left|\frac{d}{d t}\left(x_{i}(t)\right)\right| \leq \frac{\left\|a^{\prime}\right\|_{\infty}+\left\|\partial_{x} b\right\|_{\infty}\|f\|_{L^{\infty}\left(L^{1}\right)}}{C t},
$$

and $x_{i}(\cdot)$ is $\tilde{C}$-Lipschitz continuous on $[R,+\infty) \cap\left\{t ; x_{i}(t) \in \operatorname{Int}\left(I_{i}^{\delta}\right)\right\}$, with $R>0$. Moreover, we know that $x_{i}(\cdot)$ is continuous on $\mathbb{R}_{+}^{*}$ and is locally constant equal to $\bar{x}_{i}-\delta$ or $\bar{x}_{i}+\delta$ on $\left\{t ; x_{i}(t) \in \operatorname{Int}\left(I_{i}^{\delta}\right)\right\}^{c}=\left\{t ; x_{i}(t) \in \partial I_{i}^{\delta}\right\}$.

The interval $[R,+\infty)$ is connected, thus it is enough to prove that $x_{i}(\cdot)$ is locally $\tilde{C}$ Lipschitz continuous. Let $t \in[R,+\infty)$.

If $x_{i}(t) \in \operatorname{int}\left(I_{i}^{\delta}\right)$, this is true on a neighbourhood of $t$, and $x_{i}(\cdot)$ is $\tilde{C}$-Lipschitz continuous on this neighbourhood by (4.29). 
If $x_{i}(t)=\bar{x}_{i}-\delta, x_{i}$ is continuous, and on a neighbourhood of $t, x_{i}(\cdot) \leq \bar{x}_{i}$. Lemma 4.1 applies to this neighbourhood.

If $x_{i}(t)=\bar{x}_{i}+\delta$, Lemma 4.1 also applies.

Finally, $x_{i}(\cdot)$ is $\tilde{C}$-Lipschitz on $[R,+\infty)$.

2. Thank to the Lipschitz regularity obtained in part 1 of this corollary, the proof of part 3 of Proposition 4.15 can be extended to all time $t \in \mathbb{R}_{+}^{*}$, except the estimate (4.25), which must be modified in the following way :

If $x_{i}(t) \in \operatorname{Int}\left(I_{i}^{\delta}\right)$, then $\partial_{x} R\left(t, x_{i}(t)\right)=0$, and estimate (4.25) is valid.

If $x_{i}(t+h) \in \operatorname{Int}\left(I_{i}^{\delta}\right)$, then :

$$
\begin{aligned}
R\left(t, x_{i}(t+h)\right)-R\left(t, x_{i}(t)\right) & =\left(x_{i}(t+h)-x_{i}(t)\right) \partial_{x} R\left(t, x_{i}(t+h)\right)+O\left(t\left(x_{i}(t+h)-x_{i}(t)\right)^{2}\right), \\
& =O\left(t\left(x_{i}(t+h)-x_{i}(t)\right)^{2}\right),
\end{aligned}
$$

where $O\left(t\left(x_{i}(t+h)-x_{i}(t)\right)^{2}\right)$ depends on $a$ and $b$ but not on $x_{i}(t+h)$.

Finally, if $x_{i}(t), x_{i}(t+h) \notin \operatorname{Int}\left(I_{i}^{\delta}\right)$, provided that $h$ is small enough, either $x_{i}(t)=$ $x_{i}(t+h)=\bar{x}_{i}-\delta$, or $x_{i}(t)=x_{i}(t+h)=\bar{x}_{i}+\delta\left(\right.$ thanks to the regularity of $\left.x_{i}(\cdot)\right)$.

\subsection{Convergence to the Evolutionary Attractor $\left(\bar{x}_{i}\right)_{i=1, \ldots, n}$.}

Proposition 4.18 Let $a, b$ satisfy Assumption 1, and $\left(\bar{x}_{i}\right)_{i=1, \ldots, n}$ be an evolutionary attractor. There exist $\lambda_{0}, \delta_{0}>0$ (depending on $\left.a, b,\left(\bar{x}_{i}\right)_{i=1, \ldots, n}\right)$, such that if $\lambda \in\left(0, \lambda_{0}\right), \delta \in\left(0, \delta_{0}\right)$, $g_{0} \in L^{1}(X)$ satisfies Assumption 2, $g$ is the solution of (3.9) given by Theorem 3.6, $f$ is the asymptotic population distribution given by (3.11), and $\left(\bar{\rho}_{i}\right)_{i=1, \ldots, n},\left(\rho_{i}(\cdot)\right)_{i=1, \ldots, n},\left(x_{i}(\cdot)\right)_{i=1, \ldots, n}$ are defined in (2.6), (4.16), (4.19). Then

$$
\forall t>0, \forall i=1, \ldots, n, \quad x_{i}(t)=\bar{x}_{i}, \text { and } \rho_{i}(t)=\bar{\rho}_{i} .
$$

\section{Proof of Proposition 4.18.}

- We derive an evolution equation :

We know from Prop 4.17 that $\left(x_{i}(\cdot)\right)_{i=1, \ldots, n}$ is Lipschitz continuous on $[R,+\infty)$ for every $R>0$, thus $\left(x_{i}(\cdot)\right)_{i=1, \ldots, n}$ is differentiable almost everywhere. If $x_{i}(t) \in \operatorname{Int}\left(I_{i}^{\delta}\right)$, then $\frac{d}{d t} x_{i}(t)$ is given by Prop 4.16. If $x_{i}(t) \in \partial I_{i}^{\delta}$, the only possibility is $\frac{d}{d t} x_{i}(t)=0$, as $x_{i}(\cdot)$ cannot get out of $I_{i}^{\delta}$ thank to Prop 4.13. Then, for a.e. $t>0$,

$$
\begin{aligned}
\frac{d}{d t}\left\|\left(x_{i}(t)-\bar{x}_{i}\right)_{i=1, \ldots, n}\right\|^{2} & =2\left(\left(x_{i}(t)-\bar{x}_{i}\right)_{i},\left(\frac{d}{d t} x_{i}(t)\right)_{i}\right) \\
& =2 \sum_{i \in \mathcal{I}(t)}\left(x_{i}(t)-\bar{x}_{i}\right) \frac{\partial_{x} s \sum_{j=1}^{n} \rho_{j}(t) \delta_{x_{j}(t)}\left(x_{i}(t)\right)}{-\partial_{x x}^{2} R\left(t, x_{i}(t)\right)}
\end{aligned}
$$


where $\mathcal{I}(t):=\left\{i \in\{1, \ldots, n\} ; x_{i}(t) \in \operatorname{Int}\left(I_{i}^{\delta}\right)\right\}$.

Moreover, since $\partial_{x} R\left(t, x_{i}(t)\right)=0$ if $x_{i}(t) \in \operatorname{Int}\left(I_{i}^{\delta}\right)$, we have :

$$
\begin{aligned}
\sum_{i=1}^{n} \frac{\frac{d}{d t}\left(\left(x_{i}(t)-\bar{x}_{i}\right) \partial_{x} R\left(t, x_{i}(t)\right)\right)}{-\partial_{x x}^{2} R\left(t, x_{i}(t)\right)} & =\sum_{i \in \mathcal{I}(t)^{c}} \frac{\frac{d}{d t}\left(\left(x_{i}(t)-\bar{x}_{i}\right) \partial_{x} R\left(t, x_{i}(t)\right)\right)}{-\partial_{x x}^{2} R\left(t, x_{i}(t)\right)} \\
& =\sum_{i \in \mathcal{I}(t)^{c}}\left(x_{i}(t)-\bar{x}_{i}\right) \frac{\frac{d}{d t}\left(\partial_{x} R\left(t, x_{i}(t)\right)\right)}{-\partial_{x x}^{2} R\left(t, x_{i}(t)\right)}
\end{aligned}
$$

where we used the fact that if $x_{i}(t) \in \partial\left(I_{i}^{\delta}\right)$, then $\frac{d}{d t} x_{i}(t)=0$. Notice now that the derivative in time of $\partial_{x} R\left(t, x_{i}(t)\right)$ is given by :

$$
\begin{aligned}
\frac{d}{d t}\left(\partial_{x} R\left(t, x_{i}(t)\right)\right) & =\frac{d}{d t} \int_{0}^{t} \partial_{x} s \sum_{j=1}^{n} \rho_{j}(\sigma) \delta_{x_{j}(\sigma)}\left(x_{i}(t)\right) d \sigma \\
& =\partial_{x} s \sum_{j=1}^{n} \rho_{j}(t) \delta_{x_{j}(t)}\left(x_{i}(t)\right) .
\end{aligned}
$$

Thus,

$$
\sum_{i=1}^{n} \frac{\frac{d}{d t}\left(\left(x_{i}(t)-\bar{x}_{i}\right) \partial_{x} R\left(t, x_{i}(t)\right)\right)}{-\partial_{x x}^{2} R\left(t, x_{i}(t)\right)}=\sum_{i \in \mathcal{I}(t)^{c}}\left(x_{i}(t)-\bar{x}_{i}\right) \frac{\partial_{x} s \sum_{j=1}^{n} \rho_{j}(t) \delta_{x_{j}(t)}\left(x_{i}(t)\right)}{-\partial_{x x}^{2} R\left(t, x_{i}(t)\right)} .
$$

If we sum those eq. (4.30) and (4.31), we get :

$\frac{1}{2} \frac{d}{d t}\left\|\left(x_{i}(t)-\bar{x}_{i}\right)_{i=1, \ldots, n}\right\|^{2}+\sum_{i=1}^{n} \frac{\frac{d}{d t}\left(\left(x_{i}(t)-\bar{x}_{i}\right) \partial_{x} R\left(t, x_{i}(t)\right)\right)}{-\partial_{x x}^{2} R\left(t, x_{i}(t)\right)}=\sum_{i=1}^{n}\left(x_{i}(t)-\bar{x}_{i}\right) \frac{\partial_{x} s_{\sum_{j=1}^{n} \rho_{j}(t) \delta_{x_{j}(t)}}\left(x_{i}(t)\right)}{-\partial_{x x}^{2} R\left(t, x_{i}(t)\right)}$.

- We first estimate $\partial_{x x}^{2} R\left(t, x_{i}(t)\right)$ :

$$
\begin{aligned}
\partial_{x x}^{2} R\left(t, x_{i}(t)\right)= & \int_{0}^{t}\left[a^{\prime \prime}\left(x_{i}(t)\right)-\sum_{j=1}^{n} \partial_{x x}^{2} b\left(x_{i}(t), x_{j}(\sigma)\right) \rho_{j}(\sigma)\right] d \sigma \\
= & \int_{0}^{t}\left[a^{\prime \prime}\left(\bar{x}_{i}\right)-\sum_{j=1}^{n} \partial_{x x}^{2} b\left(\bar{x}_{i}, \bar{x}_{j}\right) \bar{\rho}_{j}\right] d \sigma+t O\left(\left\|x_{i}(t)-\bar{x}_{i}\right\|\right) \\
& +t O\left(\sup _{\sigma \in[0, t)}\left\|\left(x_{j}(\sigma)-\bar{x}_{j}\right)_{j=1, \ldots, n}\right\|\right)+t O\left(\sup _{\sigma \in[0, t)}\left\|\left(\rho_{j}(\sigma)-\bar{\rho}_{j}\right)_{j=1, \ldots, n}\right\|\right),
\end{aligned}
$$

and since we know from (4.28) that:

$$
\begin{aligned}
\rho_{k}(\sigma)-\bar{\rho}_{k}= & {\left[\left(b\left(x_{i}(\sigma), x_{j}(\sigma)\right)\right)_{i, j=1, \ldots, n}^{-1}\left(a\left(x_{i}(\sigma)\right)\right)_{i, j=1, \ldots, n}\right]_{k} } \\
& -\left[\left(b\left(\bar{x}_{i}, \bar{x}_{j}\right)\right)_{i, j=1, \ldots, n}^{-1}\left(a\left(\bar{x}_{i}\right)\right)_{i=1, \ldots, n}\right]_{k}, \\
= & O\left(\left\|\left(x_{j}(\sigma)-\bar{x}_{j}\right)_{j=1, \ldots, n}\right\|\right),
\end{aligned}
$$


we get the estimate:

$$
\partial_{x x}^{2} R\left(t, x_{i}(t)\right)=t \partial_{x x}^{2} s_{\sum_{j=1}^{n} \bar{\rho}_{j} \delta_{\bar{x}_{j}}}\left(\bar{x}_{i}\right)+t O\left(\sup _{\sigma \in[0, t)}\left\|\left(x_{j}(\sigma)-\bar{x}_{j}\right)_{j=1, \ldots, n}\right\|\right) .
$$

- Next, we estimate $\left(\partial_{x} s_{\sum_{j=1}^{n} \rho_{j}(t) \delta_{x_{j}(t)}}\left(x_{i}(t)\right)\right)_{i=1, \ldots, n}$.

If we take $G$ as in Definition 2.4,

$$
\begin{aligned}
\left(\partial_{x} s_{\sum_{j=1}^{n} \rho_{j}(t) \delta_{x_{j}(t)}}\left(x_{i}(t)\right)\right)_{i=1, \ldots, n}= & \left(\partial_{x} s_{\sum_{j=1}^{n} \bar{\rho}_{j} \delta_{\bar{x}_{j}}}\left(\bar{x}_{i}\right)\right)_{i=1, \ldots, n}+D G_{\left(\left(\bar{x}_{j}\right)_{j=1, \ldots, n}\right)}\left(x_{j}(t)-\bar{x}_{j}\right)_{j=1, \ldots, n} \\
& +O\left(\left\|\left(x_{j}(t)-\bar{x}_{j}\right)_{j=1, \ldots, n}\right\|^{2}\right) .
\end{aligned}
$$

Since $\left(\bar{x}_{i}\right)_{i=1, \ldots, n}$ is a singular strategy, $\partial_{x} s_{\sum_{j=1}^{n} \bar{\rho}_{j} \delta_{\bar{x}_{j}}}\left(\bar{x}_{i}\right)=0$, and thus :

$$
\left(\partial_{x} s_{\sum_{j=1}^{n} \rho_{j}(t) \delta_{x_{j}(t)}}\left(x_{i}(t)\right)\right)_{i=1, \ldots, n}=D G_{\left(\left(\bar{x}_{j}\right)_{j=1, \ldots, n}\right)}\left(x_{j}(t)-\bar{x}_{j}\right)_{j=1, \ldots, n}+O\left(\left\|\left(x_{j}(t)-\bar{x}_{j}\right)_{j=1, \ldots, n}\right\|^{2}\right) .
$$

- We use estimates (4.33), (4.34) and equality (4.32) to show a simplified inequality.

we define $F(t):=\frac{1}{2} \frac{d}{d t}\left\|\left(x_{i}(t)-\bar{x}_{i}\right)_{i=1, \ldots, n}\right\|^{2}+\sum_{i=1}^{n} \frac{\frac{d}{d t}\left(\left(x_{i}(t)-\bar{x}_{i}\right) \partial_{x} R\left(t, x_{i}(t)\right)\right)}{-\partial_{x x}^{2} R\left(t, x_{i}(t)\right)}$ to simplify notations. We see that

$$
\begin{aligned}
& F(t)=\frac{1}{t}{ }^{t}\left(x_{i}(t)-\bar{x}_{i}\right)_{i=1, \ldots, n} \\
& \operatorname{diag}\left(\left(\frac{1}{-\partial_{x x}^{2} s_{\sum_{j=1}^{n} \bar{\rho}_{j} \delta_{\bar{x}_{j}}}\left(\bar{x}_{i}\right)+O\left(\sup _{\sigma \in[0, t)}\left\|\left(x_{j}(\sigma)-\bar{x}_{j}\right)_{j=1, \ldots, n}\right\|\right)}\right)\right) \\
& \left(D G_{\left(\left(\bar{x}_{j}\right)_{j=1, \ldots, n}\right)}\left(x_{i}(t)-\bar{x}_{i}\right)_{i=1, \ldots, n}+O\left(\left\|\left(x_{i}(t)-\bar{x}_{i}\right)_{i=1, \ldots, n}\right\|^{2}\right)\right) \\
& =\frac{1}{t} t\left(x_{i}(t)-\bar{x}_{i}\right)_{i=1, \ldots, n} \operatorname{diag}\left(\left(\frac{1}{-\partial_{x x}^{2} s_{\sum_{j=1}^{n} \bar{\rho}_{j} \delta_{\bar{x}_{j}}}\left(\bar{x}_{i}\right)}+O(\delta)\right)_{i}\right) \\
& \left(D G\left(\left(\bar{x}_{j}\right)_{j=1, \ldots, n}\right)\left(x_{i}(t)-\bar{x}_{i}\right)\right)+\frac{1}{t} O\left(\left\|\left(x_{j}(t)-\bar{x}_{j}\right)_{j=1, \ldots, n}\right\|^{3}\right) .
\end{aligned}
$$

Since $\left(\bar{x}_{i}\right)_{i=1, \ldots, n}$ is a Evolutionary Attractor, we know that

$$
\forall u \in \mathbb{R}^{n}, \quad{ }^{t} u \operatorname{diag}\left(\left(\frac{1}{-\partial_{x x}^{2} s_{\sum_{j=1}^{n} \bar{\rho}_{j} \delta_{\bar{x}_{j}}\left(\bar{x}_{i}\right)}}\right)_{i=1, \ldots, n}\right) D G\left(\left(\bar{x}_{i}\right)_{i=1, \ldots, n}\right) u<-\nu\|u\|^{2}
$$

Then, provided that $\delta>0$ is small enough, 
$\forall u \in \mathbb{R}^{n}, \quad{ }^{t} u \operatorname{diag}\left(\left(\frac{1}{-\partial_{x x}^{2} s_{\sum_{j=1}^{n} \bar{\rho}_{j} \delta_{\bar{x}_{j}}}\left(\bar{x}_{i}\right)}+O(\delta)\right)_{i=1, \ldots, n}\right) D G\left(\left(\bar{x}_{i}\right)_{i=1, \ldots, n}\right) u<-\frac{3 \nu}{4}\|u\|^{2}$.

Then

$$
F(t) \leq \frac{-3 \nu}{4 t}\left\|\left(x_{i}(t)-\bar{x}_{i}\right)_{i=1, \ldots, n}\right\|^{2}+\frac{n \delta}{t} O\left(\left\|\left(x_{j}(t)-\bar{x}_{j}\right)_{j=1, \ldots, n}\right\|^{2}\right),
$$

thus, if $\delta>0$ is small enough,

$$
F(t) \leq \frac{-\nu}{2 t}\left\|\left(x_{i}(t)-\bar{x}_{i}\right)_{i=1, \ldots, n}\right\|^{2} .
$$

- We conclude from this inequality that $\forall t>0,\left(x_{i}(t)\right)_{i=1, \ldots, n}=\left(\bar{x}_{i}\right)_{i=1, \ldots, n}$ in the following way:

Integrating inequality (4.35),

$$
\begin{aligned}
\frac{1}{2}\left\|\left(x_{i}(t)-\bar{x}_{i}\right)_{i=1, \ldots, n}\right\|^{2} & +\int_{\tau}^{t} \sum_{i=1}^{n} \frac{\frac{d}{d \sigma}\left(\left(x_{i}(\sigma)-\bar{x}_{i}\right) \partial_{x} R\left(\sigma, x_{i}(\sigma)\right)\right)}{-\partial_{x x}^{2} R\left(\sigma, x_{i}(\sigma)\right)} d \sigma \\
& \leq \frac{1}{2}\left\|\left(x_{i}(\tau)-\bar{x}_{i}\right)_{i=1, \ldots, n}\right\|^{2}-\frac{\nu}{2} \int_{\tau}^{t} \frac{\left\|\left(x_{i}(\sigma)-\bar{x}_{i}\right)_{i=1, \ldots, n}\right\|^{2}}{\sigma} d \sigma \\
& \leq \frac{n}{2} \delta^{2}-\frac{\nu}{2} \int_{\tau}^{t} \frac{\left\|\left(x_{i}(\sigma)-\bar{x}_{i}\right)_{i=1, \ldots, n}\right\|^{2}}{\sigma} d \sigma
\end{aligned}
$$

Our aim is to get an estimate on $\left\|\left(x_{i}(t)-\bar{x}_{i}\right)_{i=1, \ldots, n}\right\|^{2}$ from ineq. (4.36) thanks to Gronwall's lemma. To do so, we need to find a bound from below for the term $\int_{\tau}^{t} \sum_{i=1}^{n} \frac{\frac{d}{d \sigma}\left(\left(x_{i}(\sigma)-\bar{x}_{i}\right) \partial_{x} R\left(\sigma, x_{i}(\sigma)\right)\right)}{-\partial_{x x}^{2} R\left(\sigma, x_{i}(\sigma)\right)} d$ (if it goes to $-\infty,(4.36)$ won't provide much information on $\left\|\left(x_{i}(t)-\bar{x}_{i}\right)_{i=1, \ldots, n}\right\|^{2}$ !). We use an integration by part:

$$
\begin{aligned}
& \int_{\tau}^{t} \sum_{i=1}^{n} \quad \frac{\frac{d}{d \sigma}\left(\left(x_{i}(\sigma)-\bar{x}_{i}\right) \partial_{x} R\left(\sigma, x_{i}(\sigma)\right)\right)}{-\partial_{x x}^{2} R\left(\sigma, x_{i}(\sigma)\right)} d \sigma \\
&=\sum_{i=1}^{n}\left[\frac{\left(x_{i}(\sigma)-\bar{x}_{i}\right) \partial_{x} R\left(\sigma, x_{i}(\sigma)\right)}{-\partial_{x x}^{2} R\left(\sigma, x_{i}(\sigma)\right)}\right]_{\tau}^{t} \\
& \quad-\int_{\tau}^{t} \sum_{i=1}^{n}\left(x_{i}(\sigma)-\bar{x}_{i}\right) \partial_{x} R\left(\sigma, x_{i}(\sigma)\right) \frac{d}{d \sigma}\left(\frac{1}{-\partial_{x x}^{2} R\left(\sigma, x_{i}(\sigma)\right)}\right) d \sigma \\
&=\sum_{i=1}^{n}\left[\frac{\left(x_{i}(\sigma)-\bar{x}_{i}\right) \partial_{x} R\left(\sigma, x_{i}(\sigma)\right)}{-\partial_{x x}^{2} R\left(\sigma, x_{i}(\sigma)\right)}\right]_{\tau}^{t} \\
&-\int_{\tau}^{t} \sum_{i=1}^{n}\left(x_{i}(\sigma)-\bar{x}_{i}\right) \partial_{x} R\left(\sigma, x_{i}(\sigma)\right) \frac{\partial_{x x}^{2} s_{\sum_{j=1}^{n} \rho_{j}(\sigma) \delta_{x_{j}(\sigma)}}\left(x_{i}(\sigma)\right)}{\left(\partial_{x x}^{2} R\left(\sigma, x_{i}(\sigma)\right)\right)^{2}} d \sigma .(4.37)
\end{aligned}
$$


We notice here that the last term is positive, since thanks to Remark 4.14, $\left(x_{i}(\sigma)-\right.$ $\left.\bar{x}_{i}\right) \partial_{x} R\left(\sigma, x_{i}(\sigma)\right) \geq 0$, and since $\left(\bar{x}_{i}\right)_{i}$ being an ESS, provided that $\delta>0$ is small enough, $\partial_{x x}^{2} s_{\sum_{j=1}^{n} \rho_{j}(\sigma) \delta_{x_{j}(\sigma)}}\left(x_{i}(\sigma)\right)=\partial_{x x}^{2} s_{\sum_{j=1}^{n} \bar{\rho}_{j} \delta_{\bar{x}_{j}}}\left(\bar{x}_{i}\right)+O(\delta)<0$. Then, thanks to ineq.(4.18) in Prop 4.13, (4.37) becomes :

$$
\begin{aligned}
\int_{\tau}^{t} \sum_{i=1}^{n} & \frac{\frac{d}{d \sigma}\left(\left(x_{i}(\sigma)-\bar{x}_{i}\right) \partial_{x} R\left(\sigma, x_{i}(\sigma)\right)\right)}{-\partial_{x x}^{2} R\left(\sigma, x_{i}(\sigma)\right)} d \sigma \\
& \geq \sum_{i=1}^{n}\left[\frac{\left(x_{i}(\sigma)-\bar{x}_{i}\right) \partial_{x} R\left(\sigma, x_{i}(\sigma)\right)}{-\partial_{x x}^{2} R\left(\sigma, x_{i}(\sigma)\right)}\right]_{\tau}^{t} \\
& \geq-\frac{\delta\left(\left\|a^{\prime}\right\|_{\infty}+\left\|b^{\prime}\right\|_{\infty}\|f\|_{L^{\infty}\left(M^{1}\right)}\right) t}{C t}-\frac{\delta\left(\left\|a^{\prime}\right\|_{\infty}+\left\|b^{\prime}\right\|_{\infty}\|f\|_{L^{\infty}\left(M^{1}\right)}\right) \tau}{C \tau} \\
& \geq-C,
\end{aligned}
$$

where the constant $C>0$ only depends on $a, b, \delta$. This bound from below provides an estimate on $\left\|\left(x_{i}(t)-\bar{x}_{i}\right)_{i=1, \ldots, n}\right\|^{2}$ thank to ineq. (4.36). We get :

$$
\left\|\left(x_{i}(t)-\bar{x}_{i}\right)_{i=1, \ldots, n}\right\|^{2} \leq \operatorname{Cst}(a, b, \delta)-\nu \int_{\tau}^{t} \frac{\left\|\left(x_{i}(\sigma)-\bar{x}_{i}\right)_{i=1, \ldots, n}\right\|^{2}}{\sigma} d \sigma .
$$

That is we have an inequality of the following type:

$$
\phi(t) \leq K-\nu \int_{\tau}^{t} \phi(\sigma) \psi(\sigma) d \sigma,
$$

where $\phi(t)=\left\|\left(x_{i}(t)-\bar{x}_{i}\right)_{i=1, \ldots, n}\right\|^{2}$, and $\psi(\sigma)=\frac{1}{\sigma}$. Thanks to Gronwall's Lemma, we get:

$$
\phi(t) \leq K \exp \left(-\nu \int_{\tau}^{t} \psi(\sigma) d \sigma\right)
$$

That is, for a.e. $t>0$ :

$$
\begin{aligned}
\left\|\left(x_{i}(t)-\bar{x}_{i}\right)_{i=1, \ldots, n}\right\|^{2} & \leq \operatorname{Cst}(a, b, \delta) e^{-\nu \int_{\tau}^{t} \frac{d \sigma}{\sigma}}, \\
& \leq \operatorname{Cst}(a, b, \delta)\left(\frac{\tau}{t}\right)^{\nu} .
\end{aligned}
$$

This estimate is true for a.e. $t, \tau>0$. Then, for a.e. $t>0$, we can let $\tau \rightarrow 0$ and get:

$$
\left\|\left(x_{i}(t)-\bar{x}_{i}\right)_{i=1, \ldots, n}\right\|^{2} \rightarrow 0 \text { as } \tau \rightarrow 0,
$$

which provides the result : for a.e. $t>0, x_{i}(t)=\bar{x}_{i}$.

\section{Examples and numerical simulations.}

In this section, we study numerically some explicit cases. The numerical results are obtained thanks to the numerical scheme described in [4]. 


\subsection{Examples of monomorphic ESS and Evolutionary Attractors.}

Let consider the monomorphic strategies when $a, b$ are defined by

$$
a(x)=1+A x^{2}, \quad b(x, y)=1+B(x-y)^{2}, \quad A \neq 0 .
$$

Let $\left(\bar{x}_{1}\right)$ be a monomorphic strategy, and $\bar{g}=\delta_{\bar{x}_{1}}$ the associated population (see (2.6)). This strategy $\left(\bar{x}_{1}\right)$ is singular (see Rem. 2.3) if

$$
\partial_{x} s_{\bar{g}}\left(\bar{x}_{1}\right)=2 A \bar{x}_{1}=0,
$$

that is the only singular monomorphic strategy for $a, b$ defined by (5.38) is $\bar{x}_{1}=0$. We now investigate under which conditions this singular strategy is an ESS or an Evolutionary Attractor:

- $\left(\bar{x}_{1}=0\right)$ is an ESS if :

$$
\partial_{x x}^{2} s_{\bar{g}}\left(\bar{x}_{1}\right)=2(A-B)<0,
$$

that is if and only if $A<B$.

- $\left(\bar{x}_{1}=0\right)$ is an Evolutionary Attractor if it is an ESS and for every $u \in \mathbb{R}$,

$$
u \frac{1}{-\partial_{x x}^{2} s_{\bar{g}}(0)} D G(0) u=u^{2} \frac{2 A}{-2(A-B)} \leq-\nu u^{2},
$$

for some $\nu>0$. That is, $\left(\bar{x}_{1}\right)$ is a evolutionary attractor if and only if $A<B$ and $A<0$.

We now illustrate numerically the notions of ESS and Evolutionary Attractor :

- In fig. 1 and fig. 2, we consider the case of an ESS wich is not an evolutionary attractor, that is $0<A<B$. In fig. 1, the initial condition $g_{0}$ is symmetric and then the population $g$ gathers in $x=0$. In fig. 2 , the initial condition $g_{0}$ is not symmetric and then the population $g$ gets away from $x=0$. This behaviour is typical of an ESS wich is not an evolutionary attractor.

- In fig. 3, we consider an evolutionary Attractor, that is $A<B, A<0$. The population $g$ evolves towards $\bar{x}_{1}=0$, and then gathers around that trait.

- In fig. 4 and fig. 5, we consider the case where $B<A<0$. In this situation, $\bar{x}_{i}=0$ is not an ESS, but is known in the field of adaptive dynamics as a Convergent stable strategy (CSS), (see Remark 2.5). This kind of unstable strategy is thought to be responsible for speciation (sympatric speciation). In fig. 4, we consider an initial condition wich is not centered in $x=0$, then, the population evolves toward $x=0$, as long as it is not too close to $x=0$. In fig. 5 , we consider an initial condition wich is centered in $x=0$, then, the population splits into two different species. 

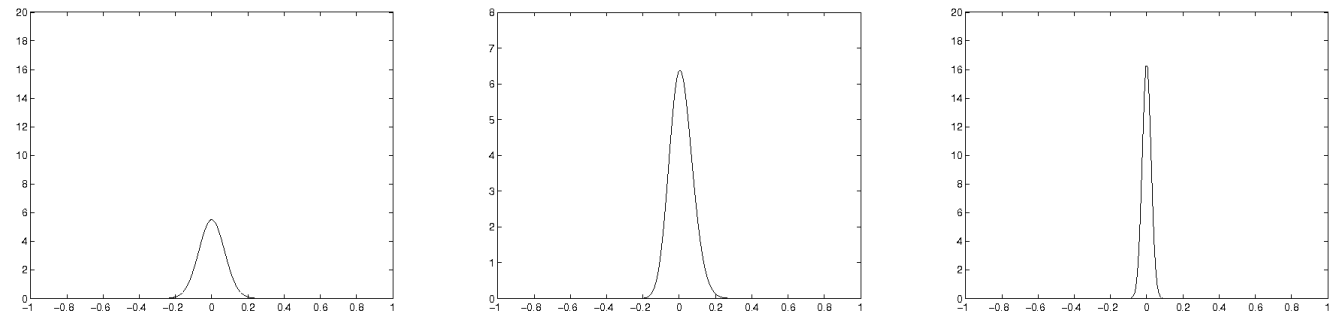

Figure 1: Simulations for $a, b$ defined by (5.38), $A=\frac{1}{2}, B=1$, and $g_{0}(x)=5.5 e^{-100 x^{2}}$, at times $t=0,500,1500$.
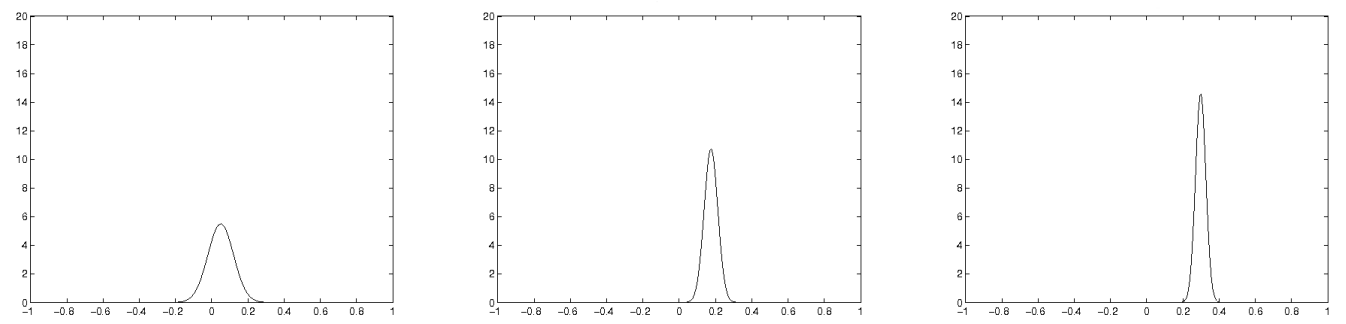

Figure 2: Simulations for $a, b$ defined by (5.38), $A=\frac{1}{2}, B=1$, and $g_{0}(x)=5.5 e^{-100(x-0.05)^{2}}$, at times $t=0,500,1000$.
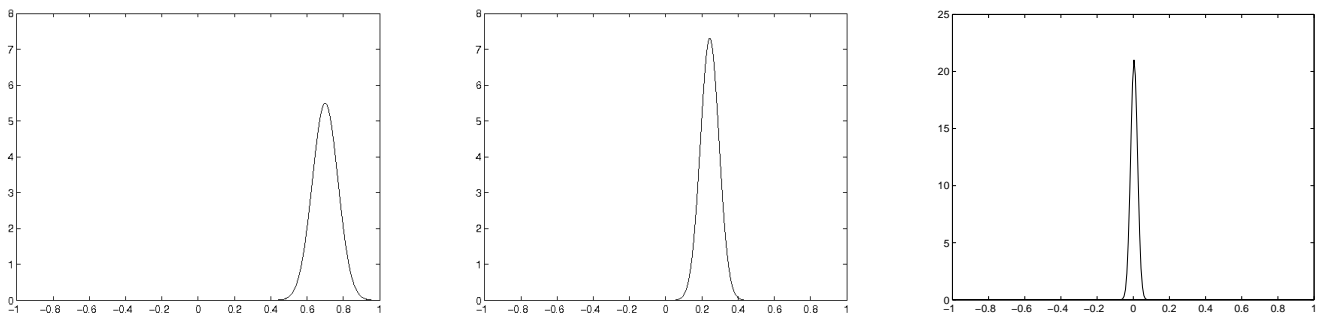

Figure 3: Simulations for $a, b$ defined by (5.38), $A=-1, B=-\frac{1}{2}$, and $g_{0}(x)=5.5 e^{-100(x-0.7)^{2}}$, at times $t=0,150,2500$. 

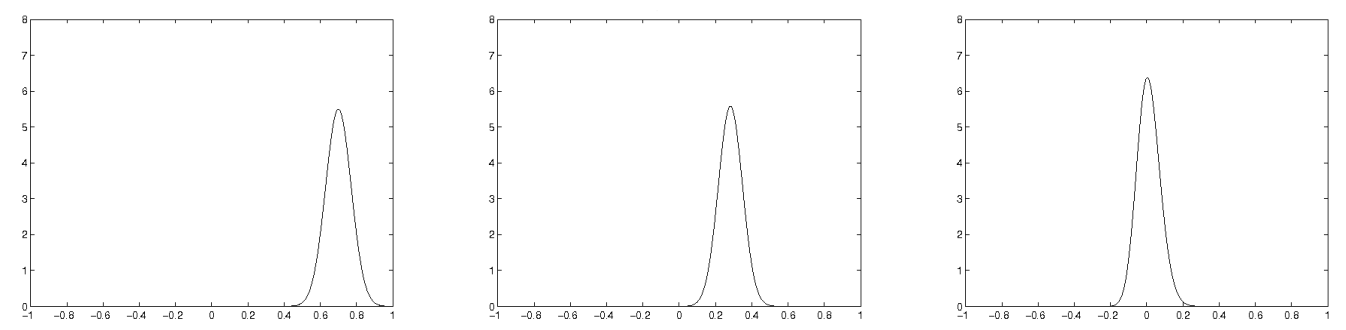

Figure 4: Simulations for $a, b$ defined by (5.38), $A=-1, B=-1.1$, and $g_{0}(x)=5.5 e^{-100(x-0.7)^{2}}$, at times $t=0,100,500$.
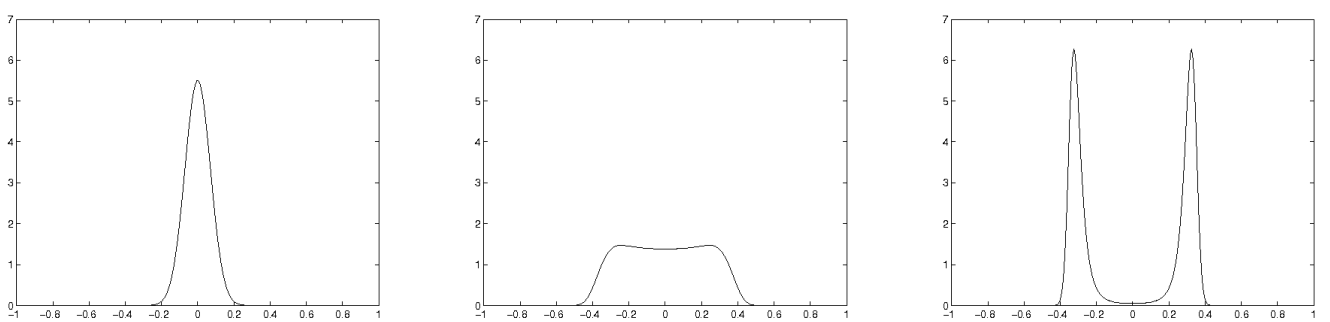

Figure 5: Simulations for $a, b$ defined by (5.38), $A=-1, B=-1.1$, and $g_{0}(x)=5.5 e^{-100 x^{2}}$, at times $t=0,850,1100$. 


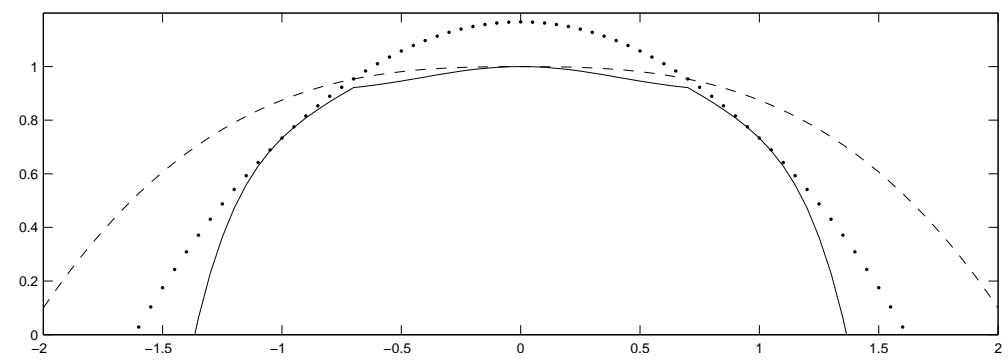

Figure 6: graphs of $a$ (continuous line), $B$ (dash line), and $\frac{2}{3}(B(\cdot+1)+B(\cdot-1))$ (dot line).

\subsection{A case where several Evolutionary Attractors exist.}

In this subsection, we construct coefficients $a$ and $b:(x, y) \mapsto B(x-y)$ such that several Evolutionary Attractors exist. If $a$ and $B$ symmetric are such that:

$$
a(1)=1, a(2)=\frac{11}{15}, B(0)=1, B(1)=\frac{7}{8}, B(2)=\frac{1}{10},
$$

then both $\bar{g}_{1}=\delta_{0}$ and $\bar{g}_{2}=\frac{2}{3}\left(\delta_{-1}+\delta_{1}\right)$ are strategies for eq. (1.1). We construct $B$ as a symmetrical spline that interpolates values (5.39), and $a$ as:

$$
a(x):=\min \left(B(x), \frac{2}{3}(B(x+1)+B(x-1))-\frac{1}{5} x^{2}(x-1)^{2}(x+1)^{2} .\right.
$$

Then, $\bar{g}_{1}=\delta_{0}$ and $\bar{g}_{2}=\frac{2}{3}\left(\delta_{-1}+\delta_{1}\right)$ are both Evolutionary Attractors for eq. (1.1), and even more:

$$
\begin{gathered}
s_{\bar{g}_{1}}(x)<0 \text { if } x \neq 0, \\
s_{\bar{g}_{2}}(x)<0 \text { if } x \notin\{-1,1\} .
\end{gathered}
$$

Numerically, we observe a local stability of each of the two Evolutionary Attractors $\bar{g}_{1}$ (see fig. 7) and $\bar{g}_{2}$ (see fig. 8).

\section{References}

[1] Calcina A., Cuadrado S., Small mutation rate and evolutionarily stable strategies in infinite dimensional adaptive dynamics, J. Math. Biol., 48, 135-159, 2004.

[2] Champagnat N., Ferrière R., Ben Arous G., The canonical equation of adaptive dynamics: A mathematical view. Selection 2, 71-81 (2001).

[3] Champagnat N., Ferrière R., Méléard S., Unifying evolutionary dynamics: From individual stochastic processes to macroscopic models. Theor. Popul. Biol. to appear.

[4] Desvillettes L., Jabin P.E., Mischler S., Raoul G., On selection dynamics for continuous populations. Preprint CMLA-ENS Cachan. 

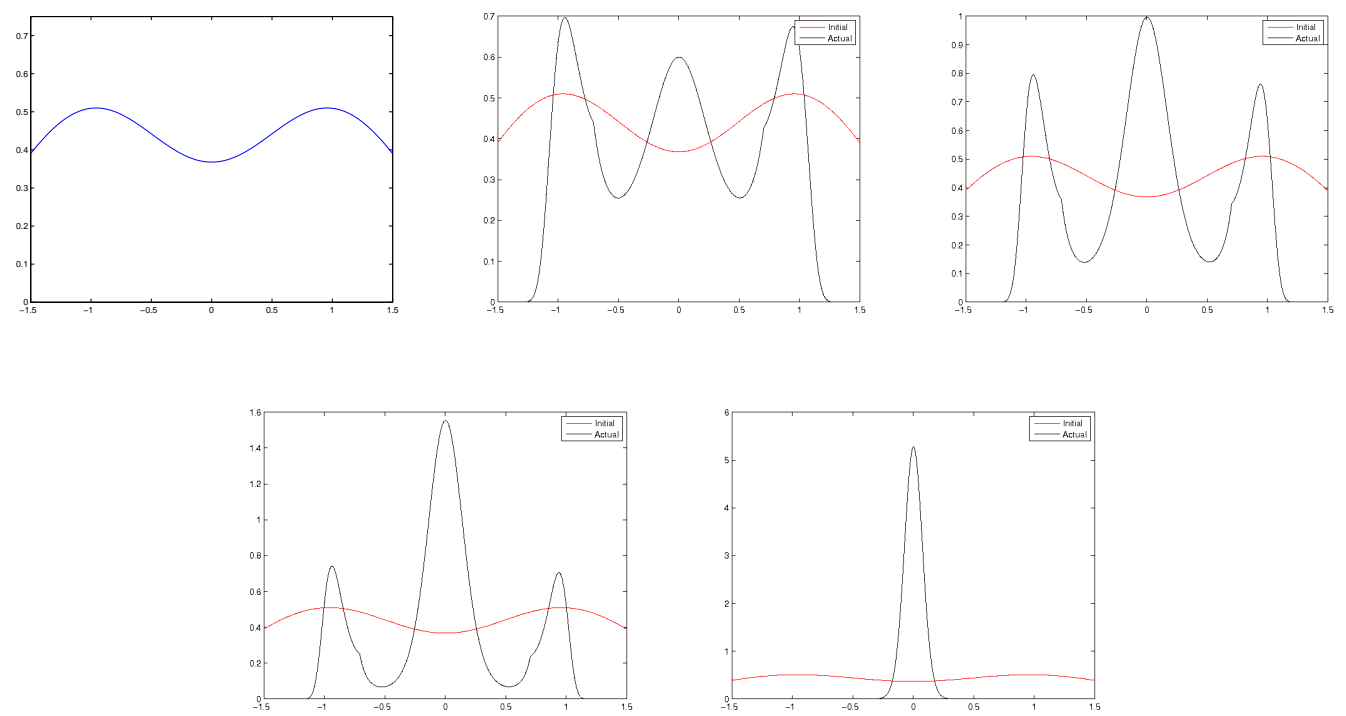

Figure 7: Simulations for $a, b$ constructed in subsection 5.2 and $g_{0}(x)=\frac{1}{2} e^{-(x+1)^{2}}+\frac{1}{2} e^{-(x-1)^{2}}$, at times $t=0,10,20,30,100$.
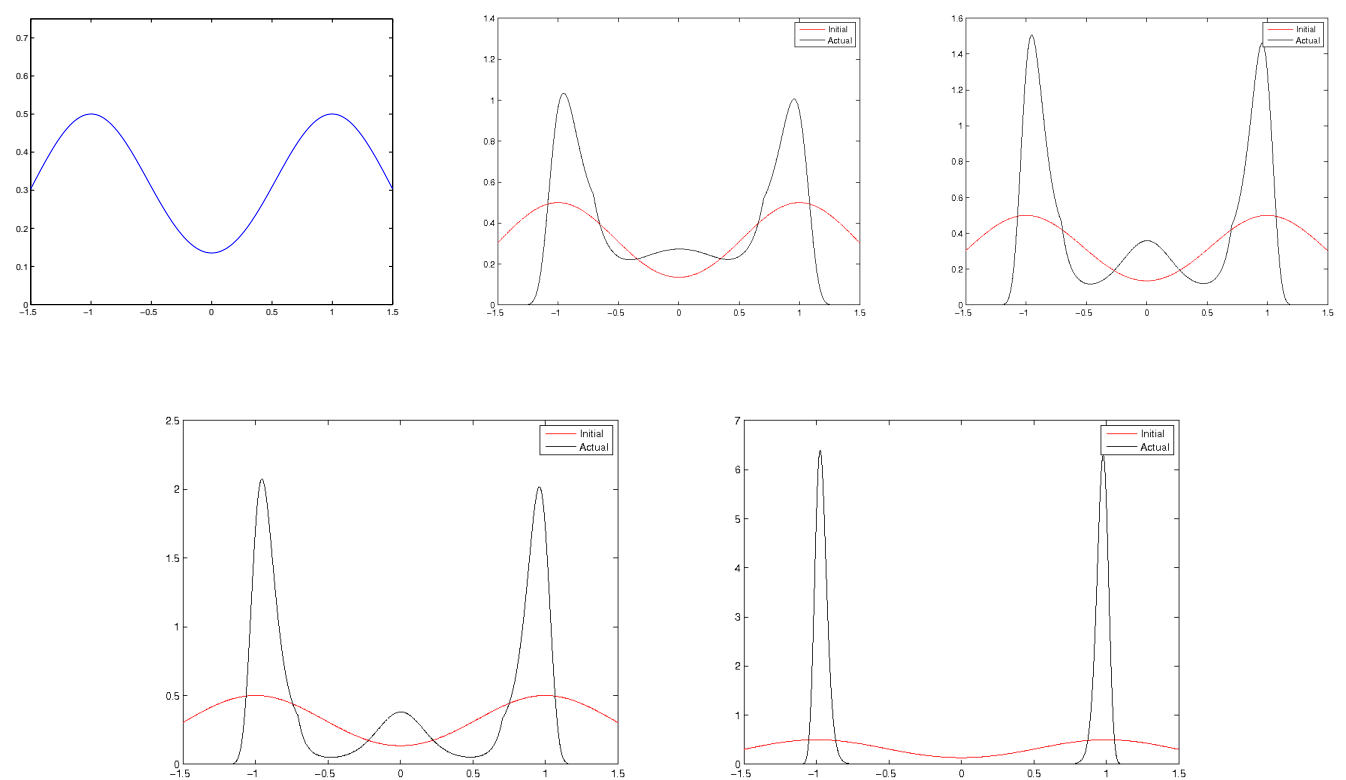

Figure 8: Simulations for $a, b$ constructed in subsection 5.2 and $g_{0}(x)=\frac{1}{2} e^{-2(x+1)^{2}}+\frac{1}{2} e^{-2(x-1)^{2}}$, at times $t=0,10,20,30,100$. 
[5] Diekmann O., Beginner's guide to adaptive dynamics, vol. 63, Banach Center Publications, $47-86$.

[6] Diekmann O., Jabin P.E., Mischler S., Perthame B., The dynamics of adaptation: An illuminating example and a Hamilton-Jacobi approach. Theor. Popul. Biol. 67, 257-271, (2005).

[7] Dieckmann U., Doebeli M., On the origin of species by sympatric speciation. Nature, 400, 354357, (1999).

[8] Dieckmann U., Law R., The dynamical theory of coevolution: a derivation from stochastic ecological processes. J. Math. Biol. 34, 579612, (1996).

[9] Geritz S. A. H., Resident-invader dynamics and the coexistence of similar strategies, J. Math. Biol., 50, 67-82, 2005.

[10] Geritz S., Kisdi E., Meszena G., Metz J., Evolutionarily singular strategies and the adaptive growth and branching of the evolutionary tree. Evolutionary Ecology, 12, 3557, (1998).

[11] Metz J. A. J., Geritz S. A. H., Mesz ńa G., Jacobs F. J. A., van Heerwaareden J. S., Adaptive dynamics: a geometrical study of the conse- quences of nearly faithful reproduction. In Stochastic and spatial structures of dynamical systems, (van Strien S. J., Lunel S. M. V., eds), pp. 183 231. KNAW Verhandelingen Amsterdam, (1996).

[12] Perthame B., Transport equation in Biology, Frontiers in Mathematics, Birkhäuser (2005).

[13] Rudin W., Real and complex analysis, Third edition, Mc Graw-Hill series in higher mathematics. Chapter 7, page 137.

[14] Waxman D., Gavrilets S., 20 questions on adaptive dynamics. Journal of Evolutionary Biology, 18, 11391154, (2005). 